\title{
SOCIALLY CONCERNED PERIODIC REVIEW REPLENISHMENT SYSTEM WITH CUSTOMER SERVICE LEVEL AND SUPPLY CHAIN CONTRACTING
}

\author{
Maryam Johari ${ }^{1}$, Seyyed-Mahdi Hosseini-Motlagh ${ }^{1, *}$, \\ Mohammadreza Nematollahi ${ }^{2}$, Mark $\mathrm{GOH}^{3}$ and Joshua Ignatius ${ }^{4}$
}

\begin{abstract}
This study contributes to the periodic review inventory system literature by: (1) investigating customer service level (CSL) as a social responsibility in pharmaceutical supply chains (PSCs) and (2) proposing supply chain contracting to achieve win-win coordination and guarantee a satisfied CSL. Specifically, considering the demand uncertainty as one of the main challenges faced by pharmacies, we analyze how applying contracts to coordinate the inventory policy of a pharmacy with that of its supplier can affect their profits and the CSL offered to society. Having shown the advantages of the centralization compared to the decentralization, we design a coordination scheme based on the quantity discount contract to encourage the players move towards centralization. Under the proposed scheme, we first obtain the minimum and maximum acceptable amounts of discount from each actor's viewpoint. Then, we propose a benefit-sharing strategy in order to make the plan interesting to both sides. The proposed discount contract and benefit-sharing strategy distribute the extra profits equitably between the two participants according to their bargaining power, which can make a win-win condition for two actors. Our results suggest that coordinating review period and order-up-to-level of the retailer and replenishment multiplier of the supplier will be of benefit in terms of both economic profitability and social responsibility.
\end{abstract}

Mathematics Subject Classification. 90B50, 90B05, 91C99.

Received September 17, 2020. Accepted March 11, 2021.

\section{INTRODUCTION}

To illuminate the importance of this study, in this section, firstly, motivation and practical background are discussed. Afterwards, theoretical background is provided. Finally, research contributions are explained.

Keywords. Supply chain coordination, quantity discount contract, customer service level, periodic review inventory policy, benefit-sharing strategy.

1 School of Industrial Engineering, Iran University of Science and Technology, Tehran, Iran.

2 School of Management Sciences, Université du Québecà, Montréal, Canada.

3 NUS Business School and The Logistics Institute - Asia Pacific, National University of Singapore, Singapore 119245, Singapore.

4 University of Exeter Business School, University of Exeter, Exeter, UK.

* Corresponding author: motlagh@iust.ac.ir 


\subsection{Motivation and practical background}

In practice, stock control is one of the important decisions taken by retailers, which indirectly impacts on the performance of the upstream supply chain members such as distributers, manufacturers, and so on. Accordingly, centralizing stock control decisions in the supply chain context can improve the performance of the whole supply chain; thus centralizing demands and supplies. In inventory systems, stock control indicates demand responsiveness of supply chain members and accordingly, it directly impacts customer satisfaction. Moreover, supply chain management is the efficient centralization of all supply chain decisions such that goods are accessible for customers. On the other hand, centralization keeps the balance between supply and demand by optimizing supply decisions from whole supply chain perspective. Therefore, centralization is the optimum decision-making framework from supply chain customers' perspective (i.e. market demand). Accordingly, investigating the centralization of supply and demand for inventory systems is of great importance which is verified by the previous literature (e.g. $[8,52])$. Accordingly, in supply chains (SCs), coordination of participants at diverse echelons (e.g. manufacturers, suppliers, and retailers) is of high importance. Moreover, sound inventory management is key to the efficiency of the retail SC, especially given the negative impact of stock-outs on customer satisfaction and the effect of stock on the economic performance of the SC entities [5-7,14]. Motivated by the importance of managing periodic review inventory system, the current research investigates the effectiveness of the periodic review ordering technique under a coordination scheme. As a remark, the periodic review $(T, R)$ setting is mainstream in the retail replenishment management literature [2]. Under this system, the stock level is evaluated every $T$ units of time and stock is ordered up to the level $R$ at each review period.

Among the coordination mechanisms, the quantity discount contract can be simply executed in contrast to the other coordination contracts. According to Weng [52], the quantity discount contracts improve the optimal safety factor of the downstream by lowering the purchase price. To this end, theoretically, some studies have used quantity discount contract as a coordination scheme [16,33,44,55,56].

As per the industrial applications, the quantity discount contract has been commonly applied in practice and can achieve SC coordination [4]. As noted by Weng [52], in practice, the quantity discount contract can be used in multiperiod ordering models where suppliers reduce the overstocking risk of retailers under quantity discount contract. On the other hand, the periodic review ordering policies are utilized in the drug pharmacies, supermarkets, and grocery stores [29]. Further, the periodic review replenishment technique $(T, R)$ is the most common and wellestablished inventory system in Iran pharmaceutical industry due to the large number of medicines.

On the other hand, in practice, providing a satisfied service level and keeping an appropriate inventory to prevent medicine shortages make pharmaceutical SC management complex. Therefore, as reported by Privett and Gonsalvez [45], using SC coordination is of great importance in the pharmaceutical SC in regard to critical and strategic role of pharmaceutical in health of society considering the complexity of pharmaceutical inventory management and demand uncertainty. As noted by Tagaras and Vlachos [51], achieving high customer service level (CSL) under the periodic review ordering technique is challengeable. However, proposing the quantity discount contract in a real-world case study of Iran pharmaceutical industry indicates that such contract is able to guarantee a satisfied service level which is of great practical application. In addition, according to the exploratory study conducted by Munson and Rosenblatt [35], the quantity discount contract is common in many industries. For instance, in the United States health care industry, many drug companies and pharmacies extensively use the quantity discount contract with reasons such as cost savings, inventory, and transportation cost [35].

\subsection{Theoretical background}

To illumine the contributions of this study, literature review is carried out based on two main streams: (1) periodic review inventory systems and (2) supply chain coordination, as the current study will be positioned in the intersection of these two research streams. This study provides a critical analysis of reviewed literature by comparing key parameters, such as customer service level factor, decision variables, type of inventory system, type of supply chain, and type of coordination contract. 
Most researches on the periodic review inventory system have been established as a single stage such as Ouyang and Chuang [43], Sarkar and Mahapatra [47], Soni and Joshi [50], and Kouki and Jouini [30]. In addition to the single-stage inventory systems, integrated inventory models have been extensively investigated under different situations by scholars such as Kanchanasuntorn and Techanitisawad [28] and Song et al. [49].

While the integrated periodic review inventory systems have attracted research and industry alike, there are policy drawbacks. For instance, implementing centralized decisions is often difficult and impractical as these decisions may financially disadvantage some SC members [21]. Accordingly, a coordination plan is needed to inspire the SC participants to make centralized choices from a SC standpoint [9, 10, 17, 18, 22-25, 36, 57].

In short, each member must have sufficient incentive to take part in the centralization. While there are many researches on the centralization of periodic review ordering model, the literature holds little on the coordination of review period and order-up-to-level choices [36]. Some of the mechanisms have been used in this domain include collaborative decision-making [36-38], credit time [13,27], and customized wholesale price [41].

As a remark, in the context of supply chain management, there exist some review papers which investigate how different approaches (e.g. supply chain contracts; information technology) affect the efficiency of supply chains. The interested readers are referred to the two review papers: (1) the review paper which is conducted by de Barros et al. [12] investigates the effects of information technology on the supply chain management, and (2) the review paper which is conducted by Guo et al. [19] investigates how channel power and channel structure influence the performance of reverse supply chains.

Johari et al. [26] propose a coordination model to analytically coordinate economic production quantity decision of a manufacturer and periodic review ordering choices of a retailer in a manufacturer-retailer SC. Nouri et al. [40] incorporate the effect of promotional effort while coordinating the periodic review ordering model. In contrast to the works by Johari et al. [26] and Nouri et al. [40], this work aims to simultaneously coordinate the replenishment multiplier decision of a supplier and periodic review replenishment decisions of a retailer by incorporating CSL into a two-level periodic review inventory system.

According to the previous literature, only Nematollahi et al. [36] investigate SC coordination under a multiechelon periodic review inventory considering service level. They develop a collaboration model to coordinate a two-level pharmaceutical SC. In their work, although the collaboration model improves SC performance relative to the decentralization, it cannot improve the SC profit up to that of the centralization (i.e. it cannot achieve full channel coordination). Moreover, their proposed collaboration model does not necessarily share the rewards between SC members equitably.

As a remark, an agent-based supply chain coordination is an effective mechanism for improving the efficiency of supply chain management. Some studies have investigated the agent-based mechanism. For instance, Giannoccaro and Pontrandolfo [15] propose a revenue-sharing scheme in and consider negotiation mechanism among SC members through an agent-based simulation method. Albino et al. [1] investigate an agent-based simulation framework in self-organized industrial symbiosis networks and propose contract mechanism to enhance the stability of industrial relationship. Meng et al. [32] analyze the effects of horizontal and vertical competition between SC members in four three-stage supply chains through a multi-agent model and various combined contracts. In addition, under contract agreements, there exit private information among supply chain members. Another mechanism which enhances the efficiency of the supply chain management is information asymmetry (information sharing) under contracts. Yao et al. [54] investigate the effect of information sharing and menu of contracts on the efficiency of the distribution channel. Ni et al. [39] investigate information asymmetry regarding the performance of the process innovation under menu of contracts. Yang et al. [53] explore the efficiency of the reverse supply chain under information sharing and contract scheme.

\subsection{Research contributions}

This study contributes to the periodic review inventory setting by extending Nematollahi et al.'s work through an employment of a quantity discount contract to achieve full channel coordination. To put it simply, the key focus of our paper is placed on the quantity discount contract to facilitate channel coordination under a periodic review ordering technique in contrast to prior works such as the study of Nematollahi et al. [36], which does not 
use coordination contracts. As noted by Li and Liu [31], the quantity discount contract can achieve full channel coordination and divide the surplus profit among channel parties based on their bargaining powers. Considering this fact, the current study applies the quantity discount contract to not only achieve full channel coordination under the periodic review ordering technique, but also to equitably divide the extra profit obtained from the coordination plan between supply chain participants based on their bargaining powers.

Moreover, the quantity discount contract is not complex and costly. However, as noted by Cachon [3], implementing the other coordination contracts (i.e. quantity-flexibility, buyback, and revenue-sharing) are more costly. In addition, the quantity discount contract has only one parameter as discount factor, which can be easily set by supplier and retailer such that channel coordination is achieved. However, as noted by Cachon [3], some coordination contracts (i.e. two-part tariff, buyback, and revenue-sharing) have a set of parameters which needs to be simultaneously adjusted to achieve channel coordination.

The proposed quantity discount contract divides the acquired benefits equitably between the SC participants. Moreover, in this study, the supplier determines the replenishment multiplier. This assumption differs from Nematollahi et al.'s model which overlooks the replenishment decisions for the upstream SC. In addition, our study considers the review period as a downstream decision in contrast to Nematollahi et al.'s model, as in practice, the buyer typically sets the review period. We develop a discount scheme to coordinate the retailer's review period and order-up-to-level, and the supplier's replenishment multiplier, so as to financially benefit both SC actors and to socially improve CSL. Finally, we develop a benefit-sharing strategy to equitably apportion the surplus SC profit between the two actors.

To sum up, the contributions of this study compared to the prior literature are: (1) suggesting a discount contract to manage supply chains with the periodic review ordering technique, (2) improving a customer service level for periodic review inventory systems by implementing a discount contract, (3) increasing the efficiency of supply chain and the profitability of all supply chain members, and (4) optimizing periodic review inventory and replenishment multiplier decisions.

The rest of the work is structured as follows. The notations and assumptions are presented in Section 2. Section 3 contains the mathematical models and solution procedures. Section 4 carries out the case study and numerical experiments for the sample data sets and a set of sensitivity analysis. Section 5 discusses concluding remarks and outlines future research paths. All proofs are provided in the Appendix A.

\section{Notations AND ASSUMptions}

Notations applied for parameters and decision variables along with assumptions used to model the SC are provided in this section.

\subsection{Notations}

\section{Parameters}

$A_{r} \quad$ Retailer ordering cost.

$A_{s} \quad$ Supplier ordering cost.

$h_{r} \quad$ Retailer inventory holding cost.

$e_{s} \quad$ Supplier purchase price.

$h_{s} \quad$ Supplier inventory holding cost.

$\vartheta \quad$ Retailer shortage cost.

$X \quad$ Protection interval $(T+L)$.

$\beta \quad$ Lost sale rate, $0 \leq \beta \leq 1$.

$X^{+} \quad \operatorname{Max}\{x, 0\}$.

$w \quad$ Wholesale price.

$p \quad$ Retail price.

$D \quad$ Mean of demand 
$L \quad$ Lead time.

$\alpha \quad$ Bargaining power of retailer.

$\sigma \quad$ Standard deviation of the demand.

\section{Decision variables}

$R$ Order-up-to-level.

$n$ Supplier replenishment multiplier.

$T$ Review period.

$k$ Safety factor.

\subsection{Assumptions}

(1) The model treats a supplier and a retailer for one item with Normally distributed demand.

(2) The level of inventory is evaluated every $T$ units of time and stock is ordered up to the level $R$, and the ordered items are received after $L$ units of time.

(3) Orders are lost if the demand cannot be met by the retailer instantly and during the shortage period, $\beta$ of the demand will be lost.

(4) Shortages are considered only for the retailer.

(5) $L$ is assumed constant and is less than $T$ i.e. there is never more than one order in any review interval.

(6) $R$ is defined as sum of the average demand throughout protection interval $(D(T+L))$ and safety stock (multiple of standard deviation of protection interval demand and safety factor) $(k \sigma \sqrt{T+L)}$ i.e. $R=$ $D(T+L)+k \sigma \sqrt{T+L}$.

\section{Model Formulations AND SOlution PROCEDURES}

This section includes three subsections. In Section 3.1, the investigated SC is modeled under the individual setting. In Section 3.2, the integrated framework for the investigated SC is discussed. Finally, Section 3.3 provides a coordinated plan for the investigated SC.

It is noteworthy that the method used in this study is "a supply chain coordination approach" as a tools of supply chain management. The coordination approach is involved three main frameworks (i.e. decentralization, centralization, and coordination) which are separately modeled in this study as decision-making structures. Moreover, the model used in this study is "a two-level supply chain with the periodic inventory system under stochastic demand". In addition, three decision variables (i.e. retailer's review period and order-up-to-level, and supplier's replenishment multiplier) are optimized in this study. To solve the optimization model, we use concavity condition and solution procedure. The purpose of using a solution procedure is that decision variables are recursive in inventory systems. In such a case, using a solution procedure under which decisions converge to the optimal values is of high importance. Figure 1 shows the methodological flow used in this study.

\subsection{Decentralized model}

A two-level multi-period SC containing a supplier and a retailer is taken into account. The periodic review ordering setting is exerted by the retailer. As a remark, review period $T$ and order-up-to-level $R$ are decision variables. The supplier delivers orders of retailer in batches and determines the replenishment multiplier, $n$. Rosenblatt and Lee [46] have shown that $n$ must be a positive integer such that it optimizes the replenishment system of supplier.

Under the decentralization, the retailer individually determines the safety factor $(k)$ and review period $(T)$ to maximize personal profit (see Eq. (3.3)) without considering the profit of supplier. Next, the supplier determines the replenishment multiplier $(n)$ by incorporating the retailer's optimal $T$ and $k$ into its profit function. Hence, the order of decisions under the decentralization is as follows:

Step 1. The retailer maximizes its profit to yield its optimal safety factor $(k)$ and review period $(T)$.

Step 2. The supplier incorporates the retailer's equilibrium solution of safety factor $(k)$ and review period $(T)$ into its profit and determines the equilibrium solution of replenishment multiplier $(n)$. 


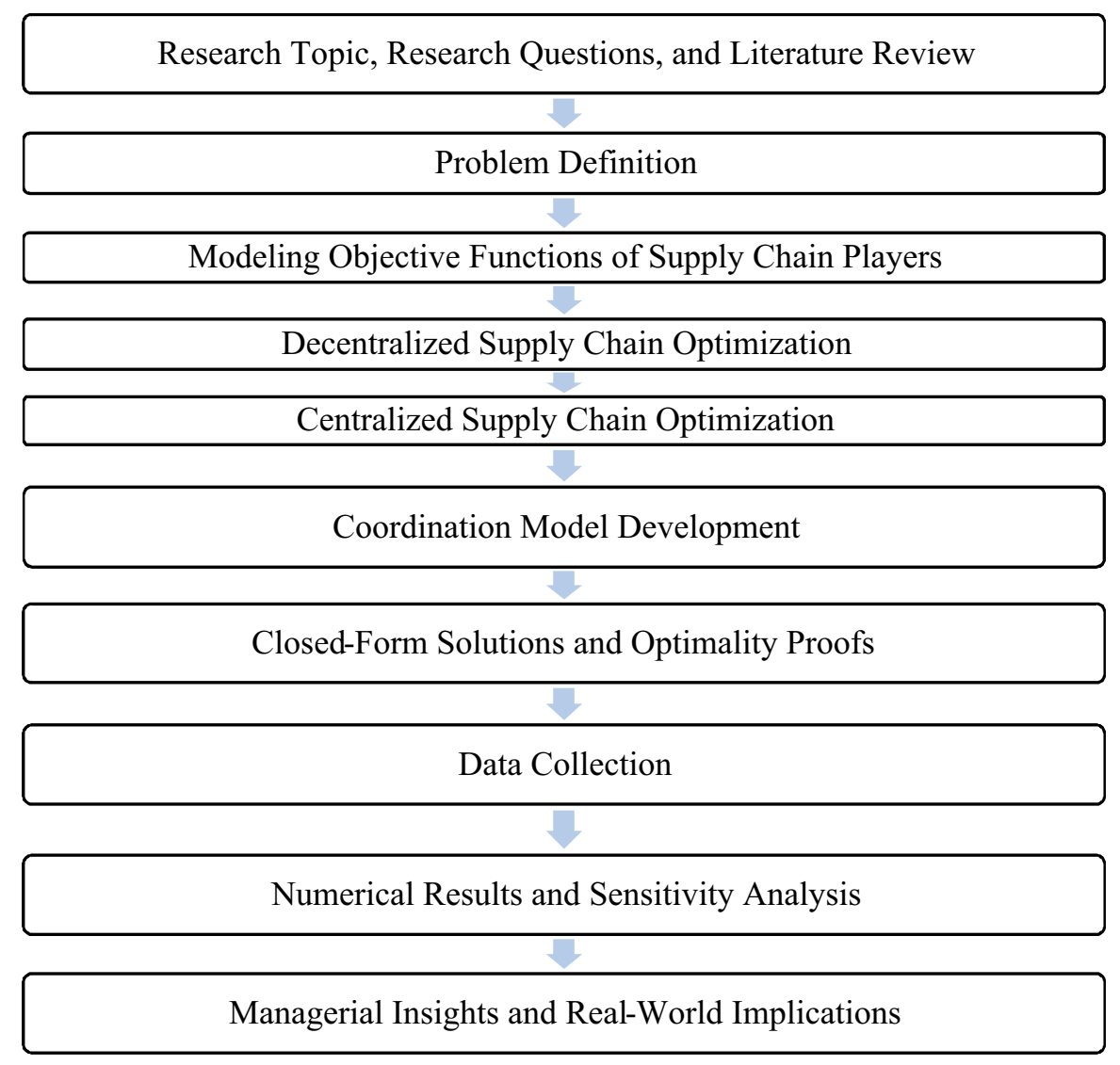

Figure 1. A methodological flow of this study.

In so doing, each member tries to maximize its own benefit separately. In the investigated SC, under the decentralized model, there are two single location inventory problems (supplier and retailer).

\subsubsection{Retailer model}

The retailer incurs costs of ordering, shortage, and holding. The cost of lost profit for each shortage unit is $(p-w)$, which forms part of the shortage cost.

The demand in the protection interval, has a Normal distribution $N(D(T+L), k \sigma \sqrt{T+L})$. $T$ is the time between the arrival and placement of two consecutive orders [34]. The average holding cost is $h_{r}\left[R-D L-\frac{D T}{2}+\beta E(X-R)^{+}\right][42]$. Further, the average stock-out cost is $\frac{\vartheta+\beta(p-w)}{T} E(X-R)^{+}$. Accordingly, the average profit of the retailer is provided in equation (3.1):

$$
\begin{aligned}
\pi_{r}(T, R)= & (p-w) D-\frac{A_{r}}{T}-h_{r}\left[R-D L-\frac{D T}{2}+\beta E(X-R)^{+}\right] \\
& -\frac{\vartheta+\beta(p-w)}{T} E(X-R)^{+} .
\end{aligned}
$$

Equation (3.1) includes received revenue and incurred costs of the retailer. The first term of equation (3.1) indicates the retailer's revenue. The ordering, holding, and stock-out costs are shown in the second, third, and fourth terms, respectively. Furthermore, the amount of average shortage $E(X-R)^{+}$at the last period of the 
cycle is shown in equation (3.2):

$$
E(X-R)^{+}=\int_{R}^{\infty}(X-R) f_{x} \mathrm{~d} x=\sigma \sqrt{T+L} \psi(k)
$$

in equation (3.2), $\Phi(k)$ and $\varphi(k)$ reflect the cumulative and standard Normal distributions, respectively and $\psi(k)=\varphi(k)-k[1-\Phi(k)]$. For simplicity, $k$ will be treated as a decision rather than $R$. Hence, the average profit of the retailer is indicated in equation (3.3):

$$
\begin{aligned}
\pi_{r}(T, k)= & (p-w) D-\frac{A_{r}}{T}-h_{r}\left[\frac{D T}{2}+k \sigma \sqrt{T+L}+\beta \sigma \sqrt{T+L} \psi(k)\right] \\
& -\frac{1}{T}(\vartheta+\beta(p-w)) \sigma \sqrt{T+L} \psi(k) .
\end{aligned}
$$

Regarding equation (3.3), the retailer should simultaneously determine $T$ and $k$.

Proposition 3.1. The profit of retailer, equation (3.3), is concave in $k$ for a given $T$.

Differentiating equation (3.3) w.r.t. $k$, the equilibrium value of $k, k^{\text {dec }}$, is determined as shown in equation (3.4):

$$
1-\Phi\left(k^{\mathrm{dec}}\right)=\frac{h_{r}}{\left[h_{r} \beta+\frac{1}{T^{\mathrm{dec}}}(\vartheta+\beta(p-w))\right]} .
$$

Equation (3.4) shows the equilibrium solution of $k$ in the decentralized framework. As a remark, $T^{\text {dec }}$ can be found from equation (3.4), i.e. $T^{\mathrm{dec}} \leq \frac{\vartheta+\beta(p-w)}{(1-\beta) h_{r}}$. Hence, the equilibrium values of $(T, k)$ as indicated by $\left(T^{\mathrm{dec}}, k^{\mathrm{dec}}\right)$ are achieved via the following solution procedure.

\section{Locally optimal solution procedure}

An efficient solution procedure that converges to $\left(T^{\mathrm{dec}}, k^{\mathrm{dec}}\right)$ is established as follows:

Step 1. Let $T^{\text {dec }}$ be the minimum feasible value.

Step 2. Calculate $k^{\text {dec }}$ using equation (3.4), considering the amount of $T^{\text {dec }}$ from Step 1.

Step 3. Calculate $\pi_{r}\left(T^{\mathrm{dec}}, k^{\mathrm{dec}}\right)$ using equation (3.3) based on the amounts of $T^{\mathrm{dec}}$ and $k^{\mathrm{dec}}$ from steps 1 and 2.

Step 4. If $T^{\text {dec }}>\frac{\vartheta+\beta(p-w)}{(1-\beta) h_{r}}$, terminate the solution procedure; else $T^{\operatorname{dec}}=T^{\mathrm{dec}}+\varepsilon$ (where $\varepsilon$ is the lowest feasible amount for $T^{\mathrm{dec}}$ ) and go to Step 2.

Step 5. Set $\left(T^{\mathrm{dec}}, k^{\mathrm{dec}}\right)$ which creates the retailer's maximum profit is optimal.

\subsubsection{Supplier model}

In the decentralized setting, the supplier determines the replenishment multiplier, $n$. As the supplier does not directly receive items from the end customer, the supplier relies on the retailer for the demand required. Due to lost sales, the demand at the supplier's level is defined as "the retailer's demand less the lost sales" as provided in equation (3.5):

$$
D-\frac{\beta}{T} \sigma \sqrt{T+L} \psi(k) .
$$

According to equation (3.5), the supplier gets the retailer's orders in fixed periods (i.e. review periods of retailer). Hence, based on the study of Chaharsooghi and Heydari [8], the supplier's annual ordering cost is $\frac{A_{s}}{n T}, n \in Z^{+}$. In addition, the expected inventory is indicated in equation (3.6):

$$
\left[\frac{(n-1)(D T-\beta \sigma \sqrt{T+L} \psi(k))}{2}\right] .
$$


Regarding equations (3.5) and (3.6), the average profit of the supplier is calculated in equation (3.7):

$$
\pi_{s}(n)=\left(w-e_{s}\right)\left(D-\frac{\beta}{T} \sigma \sqrt{T+L} \psi(k)\right)-\frac{A_{s}}{n T}-h_{s}\left[\frac{(n-1)(D T-\beta \sigma \sqrt{T+L} \psi(k))}{2}\right] .
$$

Equation (3.7) includes received revenue and incurred costs of the supplier. As a remark, the supplier's revenue is indicated in the first term. The ordering and inventory holding costs are shown in second and third terms, respectively. The supplier's average profit function depends on $T$ and $k$; however, the supplier is not able to change the retailer's decisions about the safety factor, $k$, and review period, $T$, under the decentralized structure. The supplier tries to find its decision variable, $n$, such that its profit function is optimized.

Proposition 3.2. The profit of supplier is concave in $n$.

Setting $\frac{\partial \pi_{s}(n)}{\partial n}=0$ results in equation (3.8) which yields a real value for optimal $n$. As $n$ is an integer, integer values smaller and larger than $n$ (i.e. $[n]$ and $[n]+1)$ are put in equation (3.7) and the integer that creates greater profit can be selected as $n^{\mathrm{dec}}$, which is calculated in equation (3.8):

$$
n^{\mathrm{dec}}=\sqrt{\frac{2 A_{s}}{h_{s} T^{\mathrm{dec}}\left(D T^{\mathrm{dec}}-\beta \sigma \sqrt{T^{\mathrm{dec}}+L} \psi\left(k^{\mathrm{dec}}\right)\right)}} .
$$

Equation (3.8) denotes the supplier's optimal decision in the decentralized setting which is shown by $n^{\text {dec }}$. Moreover, the retailer's optimal policy is indicated by $T^{\mathrm{dec}}$ and $k^{\mathrm{dec}}$. The average profit of the overall SC under the decentralization is provided in equation (3.9):

$$
\pi_{\mathrm{SC}}^{\mathrm{dec}}\left(T^{\mathrm{dec}}, k^{\mathrm{dec}}, n^{\mathrm{dec}}\right)=\pi_{r}\left(T^{\mathrm{dec}}, k^{\mathrm{dec}}\right)+\pi_{s}\left(n^{\mathrm{dec}}\right) .
$$

According to equation (3.9), the aggregate profit of whole SC under the decentralized setting is the sum of the supplier's and retailer's profit functions.

\subsection{Centralized model}

In this setting, all chain participants are vertically integrated under one actor orchestrating as the SC manager [8]. The manager centrally determines the SC operational decisions (i.e. $T, k$, and $n$ ) to optimize the overall SC profit. In this setting, each chain participant does not seek to maximize its own profit independently. In the investigated problem, the total profit of overall SC under the integrated setting is provided in equation (3.10):

$$
\begin{aligned}
\pi_{\mathrm{SC}}(T, k, n)= & \pi_{r}(T, k)+\pi_{s}(n)=(p-w) D-\frac{A_{r}}{T} \\
& -h_{r}\left[\frac{D T}{2}+k \sigma \sqrt{T+L}+\beta \sigma \sqrt{T+L} \psi(k)\right]-\frac{1}{T}(\vartheta+\beta(p-w)) \sigma \sqrt{T+L} \psi(k) \\
& +\left(w-e_{s}\right)\left(D-\frac{\beta}{T} \sigma \sqrt{T+L} \psi(k)\right)-\frac{A_{s}}{n T}-h_{s}\left[\frac{(n-1)(D T-\beta \sigma \sqrt{T+L} \psi(k))}{2}\right] .
\end{aligned}
$$

Simplifying equation (3.10) leads to equation (3.11):

$$
\begin{aligned}
\pi_{\mathrm{SC}}(T, k, n)= & \left(p-e_{s}\right) D-\frac{1}{T}\left[A_{r}+\frac{A_{s}}{n}\right]-\frac{\sigma \sqrt{T+L} \psi(k)}{T}\left[\left(\vartheta+\beta\left(p-e_{s}\right)\right)-\frac{h_{s}(n-1) \beta T}{2}\right] \\
& -\frac{D T}{2}\left[h_{r}+h_{s}(n-1)\right]-h_{r}[k \sigma \sqrt{T+L}+\beta \sigma \sqrt{T+L} \psi(k)] .
\end{aligned}
$$

The decision variables in this setting are $T, k, n$. 
Proposition 3.3. The profit of $S C$ is concave in $n$ and $k$ for a given $T$.

Setting $\frac{\partial \pi_{\mathrm{SC}}}{\partial T}=0, T^{\mathrm{cen}}$ is obtained as equation (3.12):

$$
\begin{aligned}
\frac{1}{T^{\mathrm{cen} 2}}\left(A_{r}+\frac{A_{s}}{n^{\mathrm{cen}}}\right)= & {\left[\left(\vartheta+\beta\left(p-e_{s}\right)\right) \sigma \psi\left(k^{\mathrm{cen}}\right)\right]\left(\frac{1}{2 T \sqrt{T^{\mathrm{cen}}+L}}-\frac{\sqrt{T^{\mathrm{cen}}+L}}{T^{\mathrm{cen}} 2}\right)-\frac{h_{s}\left(n^{\mathrm{cen}}-1\right) \beta \sigma \psi\left(k^{\mathrm{cen}}\right)}{4 \sqrt{T^{\mathrm{cen}}+L}} } \\
& +\frac{D}{2}\left[h_{r}+h_{s}\left(n^{\mathrm{cen}}-1\right)\right]+h_{r}\left[\frac{k^{\mathrm{cen}} \sigma}{2 \sqrt{T^{\mathrm{cen}}+L}}+\frac{\beta \sigma \psi\left(k^{\mathrm{cen}}\right)}{2 \sqrt{T^{\mathrm{cen}}+L}}\right] .
\end{aligned}
$$

Equation (3.12) indicates the equilibrium solution of the review period under the centralization. Also, setting $\frac{\partial \pi_{\mathrm{SC}}}{\partial k}=0, k^{\mathrm{cen}}$ can be found as equation (3.13):

$$
1-\Phi\left(k^{\mathrm{cen}}\right)=\frac{2 h_{r} T^{\mathrm{cen}}}{2\left(\vartheta+\beta\left(p-e_{s}\right)\right)+2 T^{\mathrm{cen}} \beta h_{r}-T^{\mathrm{cen}} \beta h_{s}\left(n^{\mathrm{cen}}-1\right)} .
$$

Equation (3.13) shows the equilibrium solution of the safety factor under the centralization. Likewise, with $\frac{\partial \pi_{S C}}{\partial n}=0$, the optimal value of $n$ can be found in equation (3.14):

$$
n^{\mathrm{cen}}=\sqrt{\frac{2 A_{s}}{h_{s} T^{\mathrm{cen}}\left(D T^{\mathrm{cen}}-\beta \sigma \sqrt{T^{\mathrm{cen}}+L} \psi\left(k^{\mathrm{cen}}\right)\right)}} .
$$

Equation (3.14) indicates the equilibrium solution of the replenishment multiplier under the centralization. As a remark, equations (3.13) and (3.14) are recursive. Under the centralization, the retailer's equilibrium solutions are indicated by $T^{\mathrm{cen}}$ and $k^{\mathrm{cen}}$ and the supplier's equilibrium solution is denoted by $n^{\mathrm{cen}}$. Thus, a solution procedure for finding $T^{\mathrm{cen}}, k^{\mathrm{cen}}$, and $n^{\mathrm{cen}}$ is as follows:

\section{Globally optimal solution procedure}

To specify the equilibrium solutions of $T, k$, and $n$ under the centralization, the following solution procedure is conducted. As a remark, in the proposed solution procedure, for each $T$, the optimal amounts of decisions (i.e. $k$ and $n$ ) and the profit function of entire SC are first calculated. Then, the new value for $T$ is computed based on $k$ and $n$ which are determined based on the old $T$. Accordingly, the value of $T$ is updated iteratively.

Step 1. Put $T^{\text {cen }}$ as the smallest feasible amount.

Step 2. Put $n^{\text {cen }}=1$ (smallest amount for $n$ ).

Step 3. Find $k^{\text {cen }}$ through equation (3.13).

Step 4. Find $n^{\text {cen }}$ through equation (3.14), considering the amount of $k^{\text {cen }}$ found in Step 3.

Step 5. If two consecutive amounts of $n^{\mathrm{cen}}$ are equal, then go to Step 6; else, go to Step 3.

Step 6. Compute the profit function of entire SC through equation (3.11) considering the last found amounts of $T^{\mathrm{cen}}, k^{\mathrm{cen}}$, and $n^{\mathrm{cen}}$.

Step 7. Find $T^{\mathrm{cen}}$ satisfying equation (3.12) by using a numerical search approach (update $T$ based on the computed values of $k^{\mathrm{cen}}$, and $\left.n^{\mathrm{cen}}\right)$.

Step 8. Set $\left(T^{\text {cen }}, k^{\text {cen }}, n^{\text {cen }}\right)$ which creates the greatest benefit for the overall SC is equilibrium solution.

Conducting the solution procedure, the equilibrium solutions of the decisions, $T^{\mathrm{cen}}, k^{\mathrm{cen}}$, and $n^{\mathrm{cen}}$, are determined.

Corollary 3.4. The profit of SC under the centralization is no less than that in the centralization, i.e.

$$
\pi_{\mathrm{SC}}^{\mathrm{cen}}\left(T^{\mathrm{cen}}, k^{\mathrm{cen}}, n^{\mathrm{cen}}\right) \geq \pi_{\mathrm{SC}}^{\mathrm{dec}}\left(T^{\mathrm{dec}}, k^{\mathrm{dec}}, n^{\mathrm{dec}}\right) .
$$

Equation (3.15) compares the profit function of overall SC under decentralized and centralized settings.

Corollary 3.5. The centralization does not necessarily ensure more profit for all SC participants, i.e.

$$
\pi_{r}^{\mathrm{dec}}\left(T^{\mathrm{dec}}, k^{\mathrm{dec}}\right) \geq \pi_{r}^{\mathrm{cen}}\left(T^{\mathrm{cen}}, k^{\mathrm{cen}}\right) .
$$

Equation (3.16) compares the profit of retailer under decentralization and centralization. 


\subsection{Coordination model: discount factor strategy}

As per the practical implication of coordination model in the pharmaceutical industry, Privett and Gonsalvez [45] report that lack of supply chain coordination is extracted as a critical issue in the pharmaceutical supply chain based on the interview with pharmaceutical experts. Moreover, they mention that in the pharmaceutical industry, the second challenge relates to management of inventory decisions. Accordingly, motivated by these critical issues observed in the pharmaceutical supply chain, we develop a quantity discount scheme to coordinate the pharma-retailer's periodic review replenishment and pharma-supplier's replenishment multiplier choices simultaneously. Also, as achieving high customer service level under the periodic review ordering technique is challengeable [51], conducting coordination setting is of great importance.

Clearly, as the retailer's decision would affect a supplier's profit, a decentralized solution is weaker than a centralization which works to enhance the entire SC profit relative to the decentralized setting. However, the centralization does not certainly benefit all SC actors. For instance, in the investigated problem, although $T^{\mathrm{cen}}$ and $k^{\text {cen }}$ maximize the entire SC profit, selecting $T^{\text {cen }}$ and $k^{\text {cen }}$ instead of $T^{\text {dec }}$ and $k^{\text {dec }}$ decreases the retailer's profit. This leaves the retailer with no incentive to participate in a centralization.

Hence, we propose a quantity discount scheme as a coordinated system to incentivize the retailer to change its local decisions (i.e. $T^{\mathrm{dec}}$ and $k^{\mathrm{dec}}$ ) to the global decision (i.e. $T^{\mathrm{cen}}$ and $k^{\mathrm{cen}}$ ). Indeed, the supplier by proposing a discount no worse than the difference in equation (3.16) seeks to persuade the retailer to accept joint decision-making. The parameters of the discount plan must be calibrated for both the retailer and supplier to be sufficiently motivated to participate. Thus, the supplier decreases the wholesale price from $w$ to $d w$, with $0<d<1$ as the discount factor. By implementing the operational coefficients $k_{k}$ and $k_{T}$ on the optimal safety factor and review period, respectively, the retailer shifts the review period from $T^{\text {dec }}$ to $T^{\mathrm{cen}}=k_{T} T^{\mathrm{dec}}$ and the safety factor from $k^{\text {dec }}$ to $k^{\text {cen }}=k_{k} k^{\text {dec }}$. The main challenge is to set the value of $d$ to benefit both members.

\subsubsection{Participation conditions for retailer}

The coordinated plan is accepted by the retailer if its profit under the coordinated plan is better than its individual profit in the decentralized structure i.e.

$$
\pi_{r}\left(d w, T^{\mathrm{cen}}, k^{\mathrm{cen}}\right) \geq \pi_{r}\left(w, T^{\mathrm{dec}}, k^{\mathrm{dec}}\right) .
$$

Equation (3.17) shows the situation under which the coordination plan is desirable for the retailer rather than the individual setting. As a remark, $\pi_{r}\left(d w, k_{T} T^{\mathrm{dec}}, k_{k} k^{\mathrm{dec}}\right)$ indicates the retailer's profit in the coordination, which is also denoted by $\pi_{r}^{\mathrm{co}}\left(d w, T^{\mathrm{cen}}, k^{\mathrm{cen}}\right)$. The retailer's profit in the coordination, $\pi_{r}^{\mathrm{co}}\left(d w, T^{\mathrm{cen}}, k^{\mathrm{cen}}\right)$, is formulated in equation (3.18) as:

$$
\begin{aligned}
\pi_{r}^{\mathrm{co}}\left(d w, T^{\mathrm{cen}}, k^{\mathrm{cen}}\right)= & (p-d w) D-h_{r}\left[\frac{D T^{\mathrm{cen}}}{2}+k^{\mathrm{cen}} \sigma \sqrt{T^{\mathrm{cen}}+L}+\beta \sigma \sqrt{T^{\mathrm{cen}}+L} \psi\left(k^{\mathrm{cen}}\right)\right] \\
& -\frac{A_{r}}{T^{\mathrm{cen}}}-\frac{1}{T^{\mathrm{cen}}}\left((\vartheta+\beta(p-d w)) \sigma \sqrt{T^{\mathrm{cen}}+L} \psi\left(k^{\mathrm{cen}}\right)\right) .
\end{aligned}
$$

Substituting equations (3.3) and (3.18) into equation (3.17), an upper bound for $d, d_{\max }$, is obtained as equation (3.19):

$$
\begin{aligned}
(p-d w) D- & h_{r}\left[\frac{D T^{\mathrm{cen}}}{2}+k^{\mathrm{cen}} \sigma \sqrt{T^{\mathrm{cen}}+L}+\beta \sigma \sqrt{T^{\mathrm{cen}}+L} \psi\left(k^{\mathrm{cen}}\right)\right] \\
- & \frac{A_{r}}{T^{\mathrm{cen}}}-\frac{1}{T^{\mathrm{cen}}}\left((\vartheta+\beta(p-d w)) \sigma \sqrt{T^{\mathrm{cen}}+L} \psi\left(k^{\mathrm{cen}}\right)\right) \\
\geq & (p-w) D-\frac{A_{r}}{T^{\mathrm{dec}}}-h_{r}\left[\frac{D T^{\mathrm{dec}}}{2}+k^{\mathrm{dec}} \sigma \sqrt{T^{\mathrm{dec}}+L}+\beta \sigma \sqrt{T^{\mathrm{dec}}+L} \psi\left(k^{\mathrm{dec}}\right)\right] \\
& -\frac{1}{T^{\mathrm{dec}}}(\vartheta+\beta(p-w)) \sigma \sqrt{T^{\mathrm{dec}}+L} \psi\left(k^{\mathrm{dec}}\right) .
\end{aligned}
$$


Simplifying equation (3.19) leads to equation (3.20):

$$
\begin{aligned}
d\left(w D-\frac{\beta w \sigma \sqrt{T^{\mathrm{cen}}+L} \psi\left(k_{k} k^{\mathrm{dec}}\right)}{T^{\mathrm{cen}}}\right) \leq & w D+\frac{A_{r}}{T^{\mathrm{dec}}}-\frac{A_{r}}{T^{\mathrm{cen}}} \\
& +h_{r}\left[\frac{D T^{\mathrm{dec}}}{2}+k^{\mathrm{dec}} \sigma \sqrt{T^{\mathrm{dec}}+L}+\beta \sigma \sqrt{T^{\mathrm{dec}}+L} \psi\left(k^{\mathrm{dec}}\right)-\frac{D T^{\mathrm{cen}}}{2}\right. \\
& \left.-k^{\mathrm{cen}} \sigma \sqrt{T^{\mathrm{cen}}+L}-\beta \sigma \sqrt{T^{\mathrm{cen}}+L} \psi\left(k^{\mathrm{cen}}\right)\right] \\
& +\frac{1}{T^{\mathrm{dec}}}\left[(\vartheta+\beta(p-w)) \sigma \sqrt{T^{\mathrm{dec}}+L} \psi\left(k^{\mathrm{dec}}\right)\right. \\
& \left.-\frac{1}{T^{\mathrm{cen}}}\left((\vartheta+\beta p) \sigma \sqrt{T^{\mathrm{cen}}+L} \psi\left(k^{\mathrm{cen}}\right)\right)\right]
\end{aligned}
$$

Therefore, $d_{\max }$ is calculated in equation (3.21):

$$
d_{\max } \leq M+G+O .
$$

Equation (3.21) provides satisfactory condition for the retailer. As a remark, symbols which are used in equation (3.21) are defined in equations (3.22)-(3.24) as follows:

$$
\begin{aligned}
M= & \frac{w D+\frac{A_{r}}{T^{\mathrm{dec}}}-\frac{A_{r}}{T^{\mathrm{cen}}}}{\left(w D-\frac{\beta w \sigma \sqrt{T^{\mathrm{cen}}+L} \psi\left(k^{\mathrm{cen}}\right)}{T^{\mathrm{cen}}}\right)} \\
G= & \frac{h_{r}\left[\frac{D T^{\mathrm{dec}}}{2}+k^{\mathrm{dec}} \sigma \sqrt{T^{\mathrm{dec}}+L}+\beta \sigma \sqrt{T^{\mathrm{dec}}+L} \psi\left(k^{\mathrm{dec}}\right)-\beta \sigma \sqrt{T^{\mathrm{cen}}+L} \psi\left(k^{\mathrm{cen}}\right)\right]}{\left(w D-\frac{\beta w \sigma \sqrt{T^{\mathrm{cen}}+L} \psi\left(k^{\mathrm{cen}}\right)}{T^{\mathrm{cen}}}\right)} \\
& -h_{r} \frac{\frac{D T^{\mathrm{cen}}}{2}+k^{\mathrm{cen}} \sigma \sqrt{T^{\mathrm{cen}}+L}}{\left(w D-\frac{\beta w \sigma \sqrt{T^{\mathrm{cen}}+L} \psi\left(k^{\mathrm{cen}}\right)}{T_{\mathrm{cen}}}\right)} \\
O= & \frac{\frac{1}{T^{\mathrm{dec}}}\left[(\vartheta+\beta(p-w)) \sigma \sqrt{T^{\mathrm{dec}}+L} \psi\left(k^{\mathrm{dec}}\right)\right.}{\left(w D-\frac{\beta w \sigma \sqrt{T^{\mathrm{cen}}+L} \psi\left(k^{\mathrm{cen}}\right)}{T^{\mathrm{cen}}}\right)}-\frac{\frac{1}{T^{\mathrm{cen}}}\left((\vartheta+\beta p) \sigma \sqrt{T^{\mathrm{cen}}+L} \psi\left(k^{\mathrm{cen}}\right)\right)}{\left(w D-\frac{\beta w \sigma \sqrt{T^{\mathrm{cen}}+L} \psi\left(k^{\mathrm{cen}}\right)}{T^{\mathrm{cen}}}\right)} .
\end{aligned}
$$

Hence, the amount of discount $(d)$ will be accepted by the retailer that is no more than the maximum amount found from equation (3.21).

\subsubsection{Participation conditions for supplier}

To contribute in the coordination, the supplier should shift the replenishment multiplier from $n^{\text {dec }}$ to $n^{\text {cen }}=$ $k_{n} n^{\mathrm{dec}}$. Thus, the operational coefficient for the supplier is defined as $k_{n}=n^{\mathrm{cen}} / n^{\mathrm{dec}}$. In the developed discount model, the supplier decreases the wholesale price by $(1-d)$ so as to incentivize the retailer to agree on the coordination model. The supplier offers a discount if its profit surpasses the decentralized setting i.e.

$$
\pi_{s}\left(d w, n^{\mathrm{cen}}\right) \geq \pi_{s}\left(w, n^{\mathrm{dec}}\right) .
$$

Equation (3.25) indicates the situation under which the coordination plan is satisfactory for the supplier rather than the individual setting. As a remark, $\pi_{s}\left(d w, k_{n} n^{\mathrm{dec}}\right)$ indicates the supplier's profit under the coordinated plan, which can be also denoted by $\pi_{s}^{\mathrm{co}}\left(d w, n^{\mathrm{cen}}\right)$. The supplier's profit under the coordinated plan, $\pi_{s}^{\mathrm{co}}\left(d w, n^{\mathrm{cen}}\right)$, is formulated in equation $(3.26)$ as:

$$
\pi_{s}^{\mathrm{co}}\left(d w, n^{\mathrm{cen}}\right)=\left(d w-e_{s}\right)\left(D-\frac{\beta}{T^{\mathrm{cen}}} \sigma \sqrt{T^{\mathrm{cen}}+L} \psi\left(k^{\mathrm{cen}}\right)\right)-\frac{A_{s}}{n^{\mathrm{cen}} T^{\mathrm{cen}}}
$$


1088

$$
-h_{s}\left[\frac{\left(n^{\mathrm{cen}}-1\right)\left(D T^{\mathrm{cen}}-\beta \sigma \sqrt{T^{\mathrm{cen}}+L} \psi\left(k^{\mathrm{cen}}\right)\right)}{2}\right] .
$$

Putting equations (3.7) and (3.26) into equation (3.25), a lower bound for $d, d_{\min }$, is specified as equation (3.27):

$$
\begin{aligned}
\left(d w-e_{s}\right)\left(D-\frac{\beta}{T^{\mathrm{cen}}} \sigma \sqrt{T^{\mathrm{cen}}+L} \psi\left(k^{\mathrm{cen}}\right)\right) & -\frac{A_{s}}{n^{\mathrm{cen}} T^{\mathrm{cen}}}-h_{s}\left[\frac{\left(n^{\mathrm{cen}}-1\right)\left(D T^{\mathrm{cen}}-\beta \sigma \sqrt{T^{\mathrm{cen}}+L} \psi\left(k^{\mathrm{cen}}\right)\right)}{2}\right] \\
\geq & \left(w-e_{s}\right)\left(D-\frac{\beta}{T^{\mathrm{dec}}} \sigma \sqrt{T^{\mathrm{dec}}+L} \psi\left(k^{\mathrm{dec}}\right)\right)-\frac{A_{s}}{n^{\mathrm{dec}} T^{\mathrm{dec}}} \\
& -h_{s}\left[\frac{\left(n^{\mathrm{dec}}-1\right)\left(D T^{\mathrm{dec}}-\beta \sigma \sqrt{T^{\mathrm{dec}}+L} \psi\left(k^{\mathrm{dec}}\right)\right)}{2}\right] .
\end{aligned}
$$

Simplifying equation (3.27) leads to equation (3.28):

$$
\begin{aligned}
d\left(w D-\frac{\beta w \sigma \sqrt{T^{\mathrm{cen}}+L} \psi\left(k^{\mathrm{cen}}\right)}{T^{\mathrm{cen}}}\right) \geq & \left(w-e_{s}\right)\left(D-\frac{\beta}{T^{\mathrm{dec}}} \sigma \sqrt{T^{\mathrm{dec}}+L} \psi\left(k^{\mathrm{dec}}\right)-e_{s} D\right. \\
& -\frac{h_{s}}{2}\left[\left(\left(n^{\mathrm{dec}}-1\right)\left(D T^{\mathrm{dec}}-\beta \sigma \sqrt{T^{\mathrm{dec}}+L} \psi\left(k^{\mathrm{dec}}\right)\right)\right)\right. \\
& \left.-\left(n^{\mathrm{cen}}-1\right)\left(D T^{\mathrm{cen}}-\beta \sigma \sqrt{T^{\mathrm{cen}}+L} \psi\left(k^{\mathrm{cen}}\right)\right)\right] \\
& -\frac{A_{s}}{n^{\mathrm{dec}} T^{\mathrm{dec}}}+\frac{A_{s}}{n^{\mathrm{cen}} T^{\mathrm{cen}}}-\frac{e \beta \sigma \sqrt{T^{\mathrm{cen}}+L} \psi\left(k^{\mathrm{cen}}\right)}{T^{\mathrm{cen}}} .
\end{aligned}
$$

Hence, $d_{\min }$ is determined as equation (3.29):

$$
d_{\min } \geq F+V+B+Z
$$

Equation (3.29) provides the satisfactory condition for the supplier. As a remark, symbols which are used in equation (3.29) are defined in equations (3.30)-(3.33) as follows.

$$
\begin{aligned}
& F=\frac{\left(w-e_{s}\right)\left(D-\frac{\beta}{T^{\mathrm{dec}}} \sigma \sqrt{T^{\mathrm{dec}}+L} \psi\left(k^{\mathrm{dec}}\right)\right)-\frac{A_{s}}{n^{\mathrm{dec}} T^{\mathrm{dec}}}+\frac{A_{s}}{n^{\mathrm{cen}} T^{\mathrm{cen}}}-e_{s} D}{\left(w D-\frac{\beta w \sigma \sqrt{T^{\mathrm{cen}}+L} \psi\left(k^{\mathrm{cen}}\right)}{T^{\mathrm{cen}}}\right)} \\
& V=\frac{-\frac{e_{s} \beta \sigma \sqrt{T^{\mathrm{cen}}+L} \psi\left(k^{\mathrm{cen}}\right)}{T^{\mathrm{cen}}}}{\left(w D-\frac{\beta w \sigma \sqrt{T^{\mathrm{cen}}+L} \psi\left(k^{\mathrm{cen}}\right)}{T^{\mathrm{cen}}}\right)} \\
& B=-\frac{\frac{h_{s}}{2}\left[\left(\left(n^{\mathrm{dec}}-1\right)\left(D T^{\mathrm{dec}}-\beta \sigma \sqrt{T^{\mathrm{dec}}+L} \psi\left(k^{\mathrm{dec}}\right)\right)\right]\right.}{\left(w D-\frac{\beta w \sigma \sqrt{T^{\mathrm{cen}}+L} \psi\left(k^{\mathrm{cen}}\right)}{T^{\mathrm{cen}}}\right)} \\
& Z=\frac{\frac{h_{s}}{2}\left[\left(\left(n^{\mathrm{cen}}-1\right)\left(D T^{\mathrm{cen}}-\beta \sigma \sqrt{T^{\mathrm{cen}}+L} \psi\left(k^{\mathrm{cen}}\right)\right)\right)\right]}{\left(w D-\frac{\beta w \sigma \sqrt{T^{\mathrm{cen}}+L} \psi\left(k^{\mathrm{cen}}\right)}{T^{\mathrm{cen}}}\right)} .
\end{aligned}
$$

So long as the interval $\left[d_{\min }, d_{\max }\right]$ is non-empty, the developed incentive scheme can achieve channel coordination in the SC. When $d=d_{\min }$, the retailer realizes all the gained profits of coordination, and when $d=d_{\max }$, the supplier reaps all the coordination benefits. We now propose a benefit-sharing strategy to share the extra benefits between the retailer and supplier. 


\subsubsection{Benefit-sharing strategy}

Unequitable profit sharing disrupts the participation in the coordinated program. As a remark, the bargaining power of the retailer vs. the supplier is $\alpha$ and accordingly, the supplier's bargaining power is $(1-\alpha)$. We first find the increased profit by considering channel coordination and then show how it is divided between the two participants according to their bargaining power. The value of the increased profit considering the coordination plan is given in equation (3.34):

$$
\begin{aligned}
\Delta \pi= & \pi_{\mathrm{SC}}^{\mathrm{cen}}\left(T^{\mathrm{cen}}, k^{\mathrm{cen}}, n^{\mathrm{cen}}\right)-\pi_{\mathrm{SC}}^{\mathrm{dec}}\left(T^{\mathrm{dec}}, k^{\mathrm{dec}}, n^{\mathrm{dec}}\right) \\
= & \left(p-e_{s}\right) D-\frac{1}{T^{\mathrm{cen}}}\left[A_{r}+\frac{A_{s}}{n^{\mathrm{cen}}}\right]-\frac{\sigma \sqrt{T^{\mathrm{cen}}+L} \psi\left(k^{\mathrm{cen}}\right)}{T^{\mathrm{cen}}} \\
& \times\left[\left(\vartheta+\beta\left(p-e_{s}\right)\right)-\frac{h_{s}\left(n^{\mathrm{cen}}-1\right) \beta T^{\mathrm{cen}}}{2}\right]-\frac{D T^{\mathrm{cen}}}{2}\left[h_{r}+h_{s}\left(n^{\mathrm{cen}}-1\right)\right] \\
& -h_{r}\left[k^{\mathrm{cen}} \sigma \sqrt{T^{\mathrm{cen}}+L}+\beta \sigma \sqrt{T^{\mathrm{cen}}+L} \psi\left(k^{\mathrm{cen}}\right)\right]-\left\{\left(p-e_{s}\right) D-\frac{1}{T^{\mathrm{dec}}}\left[A_{r}+\frac{A_{s}}{n^{\mathrm{dec}}}\right]\right. \\
& -\frac{\sigma \sqrt{T^{\mathrm{dec}}+L} \psi\left(k^{\mathrm{dec}}\right)}{T^{\mathrm{dec}}}\left[\left(\vartheta+\beta\left(p-e_{s}\right)\right)-\frac{h_{s}\left(n^{\mathrm{dec}}-1\right) \beta T^{\mathrm{dec}}}{2}\right] \\
& \left.-\frac{D T^{\mathrm{dec}}}{2}\left[h_{r}+h_{s}\left(n^{\mathrm{dec}}-1\right)\right]-h_{r}\left[k^{\mathrm{dec}} \sigma \sqrt{T^{\mathrm{dec}}+L}+\beta \sigma \sqrt{T^{\mathrm{dec}}+L} \psi\left(k^{\mathrm{dec}}\right)\right]\right\} .
\end{aligned}
$$

Equation (3.34) indicates how the coordination scheme achieves surplus profit for the overall SC. Based on the bargaining power of the retailer, $\alpha$, the retailer obtains $\alpha$ of the achieved profit (indicated by $\Delta \pi$ ) and accordingly, the retailer's surplus profit will be $\alpha \Delta \pi$. Moreover, the supplier's portion of achieved profit will be $(1-\alpha) \Delta \pi$. Therefore, considering retailer's share of the achieved profit, the retailer's profit in the coordinated, $\pi_{r}^{\text {co }}$, is calculated as equation (3.35). As a remark, in the coordinated scheme, superscript co is used to indicate the coordination setting.

$$
\pi_{r}^{\mathrm{co}}\left(T^{\mathrm{cen}}, k^{\mathrm{cen}}\right)=\pi_{r}^{\mathrm{dec}}\left(T^{\mathrm{dec}}, k^{\mathrm{dec}}\right)+\alpha \Delta \pi .
$$

As a remark, $\pi_{r}^{\text {co }}\left(T^{\mathrm{cen}}, k^{\mathrm{cen}}\right)$, which is the retailer's profit in the coordination, is calculated in equation (3.18) as $\pi_{r}^{\mathrm{co}}\left(T^{\mathrm{cen}}, k^{\mathrm{cen}}\right)=\pi_{r}^{\mathrm{co}}\left(d w, k_{T} T^{\mathrm{dec}}, k_{k} k^{\mathrm{dec}}\right)=(p-d w) D-$ $h_{r}\left[\frac{D T^{\mathrm{cen}}}{2}+k^{\mathrm{cen}} \sigma \sqrt{T^{\mathrm{cen}}+L}+\beta \sigma \sqrt{T^{\mathrm{cen}}+L} \psi\left(k^{\mathrm{cen}}\right)\right]-\frac{A_{r}}{T^{\mathrm{cen}}}-\frac{1}{T^{\mathrm{cen}}}\left((\vartheta+\beta(p-d w)) \sigma \sqrt{T^{\mathrm{cen}}+L} \psi\left(k^{\mathrm{cen}}\right)\right)$.

Moreover, $\pi_{r}^{\mathrm{dec}}\left(T^{\mathrm{dec}}, k^{\mathrm{dec}}\right)$, which is the retailer's profit in the decentralization, is calculated in equation $(3.3)$ as $\pi_{r}^{\text {dec }}\left(T^{\text {dec }}, k^{\text {dec }}\right)=(p-w) D-\frac{A_{r}}{T^{\text {dec }}}-h_{r}\left[\frac{D T^{\text {dec }}}{2}+k^{\operatorname{dec}} \sigma \sqrt{T^{\mathrm{dec}}+L}+\beta \sigma \sqrt{T^{\mathrm{dec}}+L} \psi\left(k^{\mathrm{dec}}\right)\right]-$ $\frac{1}{T^{\mathrm{dec}}}(\vartheta+\beta(p-w)) \sigma \sqrt{T^{\mathrm{dec}}+L} \psi\left(k^{\mathrm{dec}}\right)$.

Replacing equation (3.18) in the right side of equation (3.35) and replacing equation (3.3) in the left side of equation (3.35) lead to equation (3.36):

$$
\begin{aligned}
(p-d w) D & -h_{r}\left[\frac{D T^{\mathrm{cen}}}{2}+k^{\mathrm{cen}} \sigma \sqrt{T^{\mathrm{cen}}+L}+\beta \sigma \sqrt{T^{\mathrm{cen}}+L} \psi\left(k^{\mathrm{cen}}\right)\right] \\
& -\frac{A_{r}}{T^{\mathrm{cen}}}-\frac{1}{T^{\mathrm{cen}}}\left((\vartheta+\beta(p-d w)) \sigma \sqrt{T^{\mathrm{cen}}+L} \psi\left(k^{\mathrm{cen}}\right)\right)=(p-w) D-\frac{A_{r}}{T^{\mathrm{dec}}} \\
& -h_{r}\left[\frac{D T^{\mathrm{dec}}}{2}+k^{\mathrm{dec}} \sigma \sqrt{T^{\mathrm{dec}}+L}+\beta \sigma \sqrt{T^{\mathrm{dec}}+L} \psi\left(k^{\mathrm{dec}}\right)\right] \\
& -\frac{1}{T^{\mathrm{dec}}}(\vartheta+\beta(p-w)) \sigma \sqrt{T^{\mathrm{dec}}+L} \psi\left(k^{\mathrm{dec}}\right)+\alpha \Delta \pi .
\end{aligned}
$$


Simplifying equation (3.36) leads to equation (3.37):

$$
\begin{aligned}
-d w D+\frac{d w}{T^{\mathrm{cen}}} \beta \sigma \sqrt{T^{\mathrm{cen}}+L} \psi\left(k^{\mathrm{cen}}\right)= & -w D+\frac{A_{r}}{T^{\mathrm{cen}}}-\frac{A_{r}}{T^{\mathrm{dec}}} \\
& +h_{r}\left[\frac{D T^{\mathrm{cen}}}{2}+k^{\mathrm{cen}} \sigma \sqrt{T^{\mathrm{cen}}+L}+\beta \sigma \sqrt{T^{\mathrm{cen}}+L} \psi\left(k^{\mathrm{cen}}\right)\right] \\
& -h_{r}\left[\frac{D T^{\mathrm{dec}}}{2}+k^{\mathrm{dec}} \sigma \sqrt{T^{\mathrm{dec}}+L}+\beta \sigma \sqrt{T^{\mathrm{dec}}+L} \psi\left(k^{\mathrm{dec}}\right)\right] \\
& -\frac{1}{T^{\mathrm{cen}}}\left((\vartheta+\beta p) \sigma \sqrt{T^{\mathrm{cen}}+L} \psi\left(k^{\mathrm{cen}}\right)\right) \\
& -\frac{1}{T^{\mathrm{dec}}}(\vartheta+\beta(p-w)) \sigma \sqrt{T^{\mathrm{dec}}+L} \psi\left(k^{\mathrm{dec}}\right)+\alpha \Delta \pi .
\end{aligned}
$$

To find the value of $d$, equation (3.37) is simplified to equation (3.38) as follows:

$$
\begin{aligned}
d\left(\frac{w \beta \sigma \sqrt{T^{\mathrm{cen}}+L} \psi\left(k^{\mathrm{cen}}\right)}{T^{\mathrm{cen}}}-w D\right)= & -w D+\frac{A_{r}}{T^{\mathrm{cen}}}-\frac{A_{r}}{T^{\mathrm{dec}}} \\
& +h_{r}\left[\frac{D T^{\mathrm{cen}}}{2}+k^{\mathrm{cen}} \sigma \sqrt{T^{\mathrm{cen}}+L}+\beta \sigma \sqrt{T^{\mathrm{cen}}+L} \psi\left(k^{\mathrm{cen}}\right)\right] \\
& -h_{r}\left[\frac{D T^{\mathrm{dec}}}{2}+k^{\mathrm{dec}} \sigma \sqrt{T^{\mathrm{dec}}+L}+\beta \sigma \sqrt{T^{\mathrm{dec}}+L} \psi\left(k^{\mathrm{dec}}\right)\right] \\
& -\frac{1}{T^{\mathrm{cen}}}\left((\vartheta+\beta p) \sigma \sqrt{T^{\mathrm{cen}}+L} \psi\left(k^{\mathrm{cen}}\right)\right) \\
& -\frac{1}{T^{\mathrm{dec}}}(\vartheta+\beta(p-w)) \sigma \sqrt{T^{\mathrm{dec}}+L} \psi\left(k^{\mathrm{dec}}\right)+\alpha \Delta \pi .
\end{aligned}
$$

Accordingly, the value of $d$ is obtained as equation (3.39):

$$
\begin{aligned}
d= & \frac{-w D}{\left(\frac{w \beta \sigma \sqrt{T^{\mathrm{cen}}+L} \psi\left(k^{\mathrm{cen}}\right)}{T^{\mathrm{cen}}}-w D\right)}+\frac{\frac{A_{r}}{T^{\mathrm{cen}}}-\frac{A_{r}}{T^{\mathrm{dec}}}}{\left(\frac{w \beta \sigma \sqrt{T^{\mathrm{cen}}+L} \psi\left(k^{\mathrm{cen}}\right)}{T^{\mathrm{cen}}}-w D\right)} \\
& +\frac{h_{r}\left[\frac{D T^{\mathrm{cen}}}{2}+k^{\mathrm{cen}} \sigma \sqrt{T^{\mathrm{cen}}+L}+\beta \sigma \sqrt{T^{\mathrm{cen}}+L} \psi\left(k^{\mathrm{cen}}\right)\right]}{\left(\frac{w \beta \sigma \sqrt{T^{\mathrm{cen}}+L} \psi\left(k^{\mathrm{cen}}\right)}{T^{\mathrm{cen}}}-w D\right)} \\
& -\frac{h_{r}\left[\frac{D T^{\mathrm{dec}}}{2}+k^{\mathrm{dec}} \sigma \sqrt{T^{\mathrm{dec}}+L}+\beta \sigma \sqrt{T^{\mathrm{dec}}+L} \psi\left(k^{\mathrm{dec}}\right)\right]}{\left(\frac{w \beta \sigma \sqrt{T^{\mathrm{cen}}+L} \psi\left(k^{\mathrm{cen}}\right)}{T^{\mathrm{cen}}}-w D\right)} \\
& -\frac{\frac{1}{T^{\mathrm{cen}}}\left((\vartheta+\beta p) \sigma \sqrt{T^{\mathrm{cen}}+L} \psi\left(k^{\mathrm{cen}}\right)\right)}{\left(\frac{w \beta \sigma \sqrt{T^{\mathrm{cen}}+L} \psi\left(k^{\mathrm{cen}}\right)}{T^{\mathrm{cen}}}-w D\right)} \\
& -\frac{\frac{1}{T^{\mathrm{dec}}}(\vartheta+\beta(p-w)) \sigma \sqrt{T^{\mathrm{dec}}+L} \psi\left(k^{\mathrm{dec}}\right)}{\left(\frac{w \beta \sigma \sqrt{T^{\mathrm{cen}}+L} \psi\left(k^{\mathrm{cen}}\right)}{T^{\mathrm{cen}}}-w D\right)}+\frac{\alpha \Delta \pi}{\left(\frac{w \beta \sigma \sqrt{T^{\mathrm{cen}}+L} \psi\left(k^{\mathrm{cen}}\right)}{T^{\mathrm{cen}}}-w D\right)} .
\end{aligned}
$$

Equation (3.39) calculates the discount factor $(d)$ according to the bargaining power of each SC actor. As a remark, the value of $d$ is in the interval $\left[d_{\min }, d_{\max }\right]$.

\section{NUMERICAL EXPERIMENTS AND SENSITIVITY ANALYSES}

This study conducts sensitivity analyses in two subsections. In Section 4.1, sensitivity analyses are carried out based on test problems. Section 4.2 provides sensitivity analyses based on the real case data. 
TABLE 1. Value of parameters under test problems.

\begin{tabular}{llllllll}
\hline \hline Parameter & TP\# & TP\# & TP\# & TP\# 4 & TP\# 5 & TP\# 6 & TP\# 7 \\
\hline$A_{r}$ & 40 & 90 & 120 & 100 & 80 & 150 & 140 \\
$A_{s}$ & 80 & 110 & 90 & 75 & 100 & 70 & 130 \\
$D$ & 8000 & 30000 & 25000 & 35000 & 10000 & 15000 & 20000 \\
$h_{r}$ & 6 & 7 & 5 & 4 & 8 & 3 & 5 \\
$h_{s}$ & 3 & 4 & 2 & 1.5 & 1 & 1 & 1.5 \\
$L$ & 1 & 1.3 & 0.7 & 0.4 & 1.6 & 2 & 1 \\
$p$ & 40 & 44 & 46 & 60 & 42 & 30 & 35 \\
$w$ & 38 & 34 & 36 & 52 & 40 & 27 & 33 \\
$\vartheta$ & 1 & 0.1 & 4 & 3 & 2 & 0.5 & 0.2 \\
$\beta$ & 1 & 0.1 & 0.9 & 0.7 & 0.8 & 0.5 & 0.8 \\
$\sigma$ & 3000 & 5000 & 4500 & 5500 & 3500 & 4000 & 6000 \\
$e_{s}$ & 32 & 24 & 20 & 50 & 34 & 20 & 28 \\
$\alpha$ & 0.4 & 0.5 & 0.4 & 0.6 & 0.3 & 0.1 & 0.9 \\
\hline
\end{tabular}

\subsection{Test problems}

As a remark, data of parameters in Table 1 are generated based on the wide range of parameters to assess the efficiency of the developed contract. It is noteworthy that in the literature on supply chain coordination, to demonstrate the efficiency of the contract, a wide range of parameters are carried out, through which the optimal decisions and profits of supply chain members are analyzed. The data of parameters are generated based on the experts' opinions such that they are according to the assumptions and market situation. For instance, retail price (i.e. p) should be greater than the wholesale price (i.e. $w)$, and purchase cost of supplier (i.e. $e_{s}$ ) should be less than the wholesale price (i.e. $w$ ). The data for seven test problems are provided in Table 1. Using the test problems, our model is evaluated under decentralization, centralization, and coordination settings.

Table 2 compares different decision models for the seven test problems. Analyzing the SC profit under the decentralization and centralization indicates that centralization improves the CSL, safety factor, and the SC profit. Although shifting the values of decisions from the decentralized to those of the centralized setting enhances SC profit, this change also decreases the retailer's profit (see Tab. 2). Hence, centralization is infeasible since the retailer has no incentive to accept the centralization. Thus, applying a discount mechanism as an incentive strategy facilitates channel coordination by sharing the profit between the members equitably, resulting in more profit for each member in all tests (see Tab. 2). In addition, the SC profit and amounts of the decisions in the coordinated system are equal to those of the centralization.

Considering the profit-sharing scheme, the surplus profit achieved by the channel coordination, will be divided between the participants based on their bargaining power. As shown in Table 1, the bargaining power of the retailer $(\alpha)$ is set to 0.5 in $\mathrm{TP} \# 2$. In this setting, two participants share the gains from $\mathrm{SC}$ coordination equally. From Table 2, the improvement in the retailer's and supplier's profitability through SC coordination is $285184.4-284713.6=470.8$ and $294000.9-293530.1=470.8$, respectively. This equal allocation of profit to both players shows the fairness of the coordination model.

Moreover, CSL is used to evaluate the SC performance [20]. We adopt the CSL definition from Silver and Bischak [48]. Therefore, CSL is formulated in equation (4.1) as:

$$
\mathrm{CSL}=1-\frac{\sigma \sqrt{T+L} \psi(k)}{D T} .
$$

From Table 2, the proposed discount contract enhances the CSL compared to the decentralization. Thus, the proposed discount scheme is useful when the CSL is critical. 
TABLE 2. Comparing decision models under test problems.

\begin{tabular}{|c|c|c|c|c|c|c|c|c|}
\hline \multirow{2}{*}{$\begin{array}{l}\text { Test } \\
\text { problem }\end{array}$} & \multirow[t]{2}{*}{ Item } & \multirow{2}{*}{$\begin{array}{l}\text { Decentralized } \\
\text { SC }\end{array}$} & \multirow{2}{*}{$\begin{array}{l}\text { Centralized } \\
\text { SC }\end{array}$} & \multirow{2}{*}{$\begin{array}{l}\text { Coordinated } \\
\text { SC }\end{array}$} & \multirow{2}{*}{$\begin{array}{l}\text { Increase } \\
\text { in profit }\end{array}$} & \multicolumn{3}{|c|}{ Discount factor } \\
\hline & & & & & & $d_{\min }$ & $d_{\max }$ & $d_{\text {shared }}$ \\
\hline \multirow{7}{*}{1} & $T$ & 7.24 & 6.30 & 6.30 & \multirow{7}{*}{997.40} & \multirow{7}{*}{0.99518} & \multirow{7}{*}{0.99850} & \multirow{7}{*}{0.99717} \\
\hline & $k$ & 1.77 & 2.27 & 2.27 & & & & \\
\hline & $n$ & 4 & 5 & 5 & & & & \\
\hline & $\pi_{r}$ & 7629.60 & 7179.60 & 8028.60 & & & & \\
\hline & $\pi_{s}$ & 44223.30 & 45670.70 & 44821.70 & & & & \\
\hline & $\pi_{\mathrm{SC}}$ & 51852.90 & 52850.30 & 52850.30 & & & & \\
\hline & CSL & $95.66 \%$ & $98.78 \%$ & $98.78 \%$ & & & & \\
\hline \multirow{7}{*}{2} & $T$ & 9.23 & 8.75 & 8.75 & \multirow{7}{*}{941.70} & \multirow{7}{*}{0.99853} & \multirow{7}{*}{0.99945} & \multirow{7}{*}{0.99899} \\
\hline & $k$ & 1 & 1.41 & 1.41 & & & & \\
\hline & $n$ & 2 & 2 & 2 & & & & \\
\hline & $\pi_{r}$ & 284713.60 & 284156.80 & 285184.40 & & & & \\
\hline & $\pi_{s}$ & 293530.10 & 295028.50 & 294000.90 & & & & \\
\hline & $\pi_{\mathrm{SC}}$ & 578243.70 & 579185.30 & 579185.30 & & & & \\
\hline & CSL & $90.69 \%$ & $95.85 \%$ & $95.85 \%$ & & & & \\
\hline \multirow{7}{*}{3} & $T$ & 8.98 & 9.64 & 9.64 & \multirow{7}{*}{498.10} & & & \\
\hline & $k$ & 2.35 & 2.59 & 2.59 & & & & \\
\hline & $n$ & 2 & 2 & 2 & & & & \\
\hline & $\pi_{r}$ & 233740.20 & 233429.40 & 233939.50 & & 0.99910 & 0.99965 & 0.99943 \\
\hline & $\pi_{s}$ & 396203.40 & 397012.30 & 396502.30 & & & & \\
\hline & $\pi_{\mathrm{SC}}$ & 629943.60 & 630441.70 & 630441.70 & & & & \\
\hline & CSL & $99.62 \%$ & $99.83 \%$ & $99.83 \%$ & & & & \\
\hline & $T$ & 7.91 & 8.88 & 8.88 & & & & \\
\hline & $k$ & 2.33 & 2.34 & 2.34 & & & & \\
\hline & $n$ & 3 & 2 & 2 & & & & \\
\hline 4 & $\pi_{r}$ & 265022.40 & 264961.80 & 265062.10 & 66.30 & 0.99993 & 0.99997 & 0.99994 \\
\hline & $\pi_{s}$ & 67529.80 & 67656.60 & 67556.30 & & & & \\
\hline & $\pi_{\mathrm{SC}}$ & 332552.10 & 332618.40 & 332618.40 & & & & \\
\hline & CSL & $99.63 \%$ & $99.66 \%$ & $99.66 \%$ & & & & \\
\hline & $T$ & 9.09 & 7.85 & 7.85 & & & & \\
\hline & $k$ & 1.62 & 2.05 & 2.05 & & & & \\
\hline & $n$ & 6 & 7 & 7 & & & & \\
\hline 5 & $\pi_{r}$ & 6024.40 & 5462.80 & 6338.70 & 1047.50 & 0.99591 & 0.99857 & 0.99778 \\
\hline & $\pi_{s}$ & 56154.20 & 57763.40 & 56887.50 & & & & \\
\hline & $\pi_{\mathrm{SC}}$ & 62178.70 & 63226.10 & 63226.10 & & & & \\
\hline & CSL & $94.62 \%$ & $98.05 \%$ & $98.05 \%$ & & & & \\
\hline & $T$ & 21.52 & 19.33 & 19.33 & & & & \\
\hline & $k$ & 1.37 & 1.90 & 1.90 & & & & \\
\hline & $n$ & 2 & 2 & 2 & & & & \\
\hline 6 & $\pi_{r}$ & 35547.64 & 35039.84 & 35657.65 & 1100.15 & 0.99600 & 0.99873 & 0.99846 \\
\hline & $\pi_{s}$ & 1011635.03 & 103242.98 & 102625.17 & & & & \\
\hline & $\pi_{\mathrm{SC}}$ & 137182.68 & 138282.83 & 138282.83 & & & & \\
\hline & CSL & $95.54 \%$ & $98.66 \%$ & $98.66 \%$ & & & & \\
\hline & $T$ & 12.51 & 10.87 & 10.87 & & & & \\
\hline & $k$ & 1.34 & 1.95 & 1.95 & & & & \\
\hline & $n$ & 3 & 3 & 3 & & & & \\
\hline 7 & $\pi_{r}$ & 23741.61 & 22567.29 & 26299.61 & 2842.22 & 0.99382 & 0.99819 & 0.99426 \\
\hline & $\pi_{s}$ & 92255.17 & 96271.71 & 92539.39 & & & & \\
\hline & $\pi_{\mathrm{SC}}$ & 115996.78 & 118839.00 & 118839.00 & & & & \\
\hline & CSL & $93.11 \%$ & $98.25 \%$ & $98.25 \%$ & & & & \\
\hline
\end{tabular}


TABLE 3. Comparing decisions under decision models over various $\sigma, \vartheta, h_{r}, \beta$.

\begin{tabular}{|c|c|c|c|c|c|c|c|c|c|c|c|c|}
\hline \multirow[t]{2}{*}{ Parameter } & \multirow[t]{2}{*}{ Value } & \multicolumn{4}{|c|}{ Decentralized SC } & \multicolumn{4}{|c|}{ Coordinated SC } & \multicolumn{3}{|c|}{ Discount factor } \\
\hline & & $T$ & $k$ & $n$ & CSL & $T$ & $k$ & $n$ & CSL & $d_{\min }$ & $d_{\max }$ & $d_{\text {shared }}$ \\
\hline \multirow[t]{8}{*}{$\sigma$} & 500 & 10 & 1.57 & 5 & $99.19 \%$ & 10 & 1.95 & 5 & $99.68 \%$ & 0.9994 & 0.9998 & 0.9997 \\
\hline & 1000 & 10 & 1.57 & 5 & $98.38 \%$ & 10 & 1.95 & 5 & $99.37 \%$ & 0.9988 & 0.9996 & 0.9993 \\
\hline & 1500 & 10 & 1.57 & 5 & $97.57 \%$ & 10 & 1.95 & 5 & $99.05 \%$ & 0.9982 & 0.9994 & 0.9990 \\
\hline & 2000 & 10 & 1.57 & 5 & $96.76 \%$ & 10 & 1.95 & 5 & $98.73 \%$ & 0.9976 & 0.9992 & 0.9987 \\
\hline & 2500 & 10 & 1.57 & 5 & $95.96 \%$ & 9.22 & 1.98 & 6 & $98.48 \%$ & 0.9970 & 0.9989 & 0.9983 \\
\hline & 3000 & 9.7 & 1.58 & 5 & $95.22 \%$ & 8.62 & 2.01 & 6 & $98.24 \%$ & 0.9964 & 0.9987 & 0.9980 \\
\hline & 3500 & 9.1 & 1.62 & 6 & $94.62 \%$ & 7.85 & 2.05 & 7 & $98.05 \%$ & 0.9959 & 0.9986 & 0.9978 \\
\hline & 4000 & 8.5 & 1.65 & 6 & $94.07 \%$ & 7.41 & 2.07 & 7 & $97.84 \%$ & 0.9955 & 0.9984 & 0.9975 \\
\hline \multirow[t]{9}{*}{$\vartheta$} & 1 & 9.8 & 1.42 & 5 & $91.94 \%$ & 8.23 & 1.98 & 6 & $97.70 \%$ & 0.9930 & 0.9978 & 0.9964 \\
\hline & 1.5 & 9.4 & 1.53 & 6 & $93.53 \%$ & 7.91 & 2.02 & 7 & $97.90 \%$ & 0.9948 & 0.9983 & 0.9972 \\
\hline & 2 & 9.1 & 1.625 & 6 & $94.62 \%$ & 7.85 & 2.05 & 7 & $98.05 \%$ & 0.9959 & 0.9986 & 0.9978 \\
\hline & 2.5 & 8.8 & 1.69 & 6 & $95.42 \%$ & 7.79 & 2.07 & 7 & $98.18 \%$ & 0.9967 & 0.9988 & 0.9982 \\
\hline & 3 & 8.6 & 1.75 & 6 & $96.02 \%$ & 7.74 & 2.10 & 7 & $98.29 \%$ & 0.9973 & 0.9990 & 0.9985 \\
\hline & 3.5 & 8.5 & 1.81 & 6 & $96.49 \%$ & 7.69 & 2.12 & 7 & $98.39 \%$ & 0.9977 & 0.9991 & 0.9987 \\
\hline & 4 & 8.3 & 1.86 & 6 & $96.86 \%$ & 7.65 & 2.14 & 7 & 98.4 & 0.9981 & 0.9992 & 0.9989 \\
\hline & 4.5 & 8.2 & 1.90 & 6 & $97.17 \%$ & 7.61 & 2.17 & 7 & $98.56 \%$ & 0.9983 & 0.9993 & 0.9990 \\
\hline & 5 & 8.1 & 1.94 & 6 & $97.42 \%$ & 7.57 & 2.18 & 7 & $98.64 \%$ & 0.9986 & 0.9994 & 0.9992 \\
\hline \multirow[t]{8}{*}{$h_{r}$} & 1 & 10 & 2.43 & 5 & $99.43 \%$ & 10 & 2.72 & 5 & $99.77 \%$ & 0.9996 & 0.9999 & 0.9998 \\
\hline & 2 & 10 & 2.17 & 5 & $98.79 \%$ & 10 & 2.48 & 5 & $99.52 \%$ & 0.9991 & 0.9997 & 0.9995 \\
\hline & 3 & 10 & 2.01 & 5 & $98.10 \%$ & 10 & 2.34 & 5 & $99.25 \%$ & 0.9986 & 0.9995 & 0.9992 \\
\hline & 4 & 10 & 1.89 & 5 & $97.38 \%$ & 10 & 2.23 & 5 & $98.97 \%$ & 0.9981 & 0.9993 & 0.9990 \\
\hline & 5 & 10 & 1.79 & 5 & $96.65 \%$ & 10 & 2.14 & 5 & $98.68 \%$ & 0.9975 & 0.9991 & 0.9987 \\
\hline & 6 & 10 & 1.71 & 5 & $95.89 \%$ & 9.14 & 2.10 & 6 & $98.46 \%$ & 0.9969 & 0.9989 & 0.9983 \\
\hline & 7 & 9.7 & 1.65 & 5 & $95.21 \%$ & 8.57 & 2.07 & 6 & $98.24 \%$ & 0.9964 & 0.9987 & 0.9980 \\
\hline & 8 & 9.1 & 1.62 & 6 & $94.62 \%$ & 7.85 & 2.05 & 7 & $98.05 \%$ & 0.9959 & 0.9986 & 0.9978 \\
\hline \multirow[t]{10}{*}{$\beta$} & 0.1 & 10 & 1.29 & 5 & $89.39 \%$ & 10 & 1.42 & 5 & $92.01 \%$ & 0.9996 & 0.9998 & 0.9998 \\
\hline & 0.2 & 10 & 1.34 & 5 & $90.54 \%$ & 9.77 & 1.57 & 5 & $94.16 \%$ & 0.9989 & 0.9995 & 0.9993 \\
\hline & 0.3 & 10 & 1.3 & 5 & 91.4 & 8.97 & 1.70 & 6 & $95.60 \%$ & 0.9982 & 0.9993 & 0.9989 \\
\hline & 0.4 & 9.9 & 1.44 & 5 & $92.30 \%$ & 8.70 & 1.80 & 6 & $96.44 \%$ & 0.9975 & 0.9991 & 0.9986 \\
\hline & 0.5 & 9.6 & 1.49 & 5 & $93.04 \%$ & 8.50 & 1.87 & 6 & $97.02 \%$ & 0.9970 & 0.9989 & 0.9983 \\
\hline & 0.6 & 9.4 & 1.54 & 6 & $93.66 \%$ & 8.34 & 1.93 & 6 & $97.44 \%$ & 0.9966 & 0.9988 & 0.9981 \\
\hline & 0.7 & 9.2 & 1.58 & 6 & $94.18 \%$ & 8.21 & 1.99 & 6 & $97.76 \%$ & 0.9962 & 0.9987 & 0.9979 \\
\hline & 0.8 & 9.1 & 1.62 & 6 & $94.62 \%$ & 7.85 & 2.05 & 7 & $98.05 \%$ & 0.9959 & 0.9986 & 0.9978 \\
\hline & 0.9 & 9 & 1.65 & 6 & $95.01 \%$ & 7.76 & 2.09 & 7 & $98.25 \%$ & 0.9956 & 0.9985 & 0.9976 \\
\hline & 1 & 8.8 & 1.68 & 6 & $95.34 \%$ & 7.68 & 2.13 & 7 & $98.41 \%$ & 0.9954 & 0.9984 & 0.9975 \\
\hline
\end{tabular}

As a remark, in the investigated periodic review inventory system, sensitivity analyses are provided based on the modification of key parameters (i.e. holding cost $\left(h_{r}\right)$, shortage cost $(\vartheta)$, lost sale rate $(\beta)$, and demand uncertainty $(\sigma)$ ) which affect inventory system. Specifically, to evaluate how key parameters influence the performance of the periodic inventory system, we analyze their effects on the decision variables and profits of supply chain participants, whole supply chain profit, customer service level, and discount factor. The range of key parameters are considered as $\sigma \in(500,4000), \vartheta \in(1,5), h_{r} \in(1,8), \beta \in(0.1,1)$. Table 3 is provided based on the modification of the key parameters to explore how customer service level and discount factor are affected by these key parameters. Table 4 is also provided based on the modification of the key parameters to explore how profit of supply chain and its members are affected by these key parameters. In addition to Tables 3 and 4 , some figures (Figs. 1-6) are carried out to schematically explore the efficiency of the developed contract based on the modification of key parameters. 


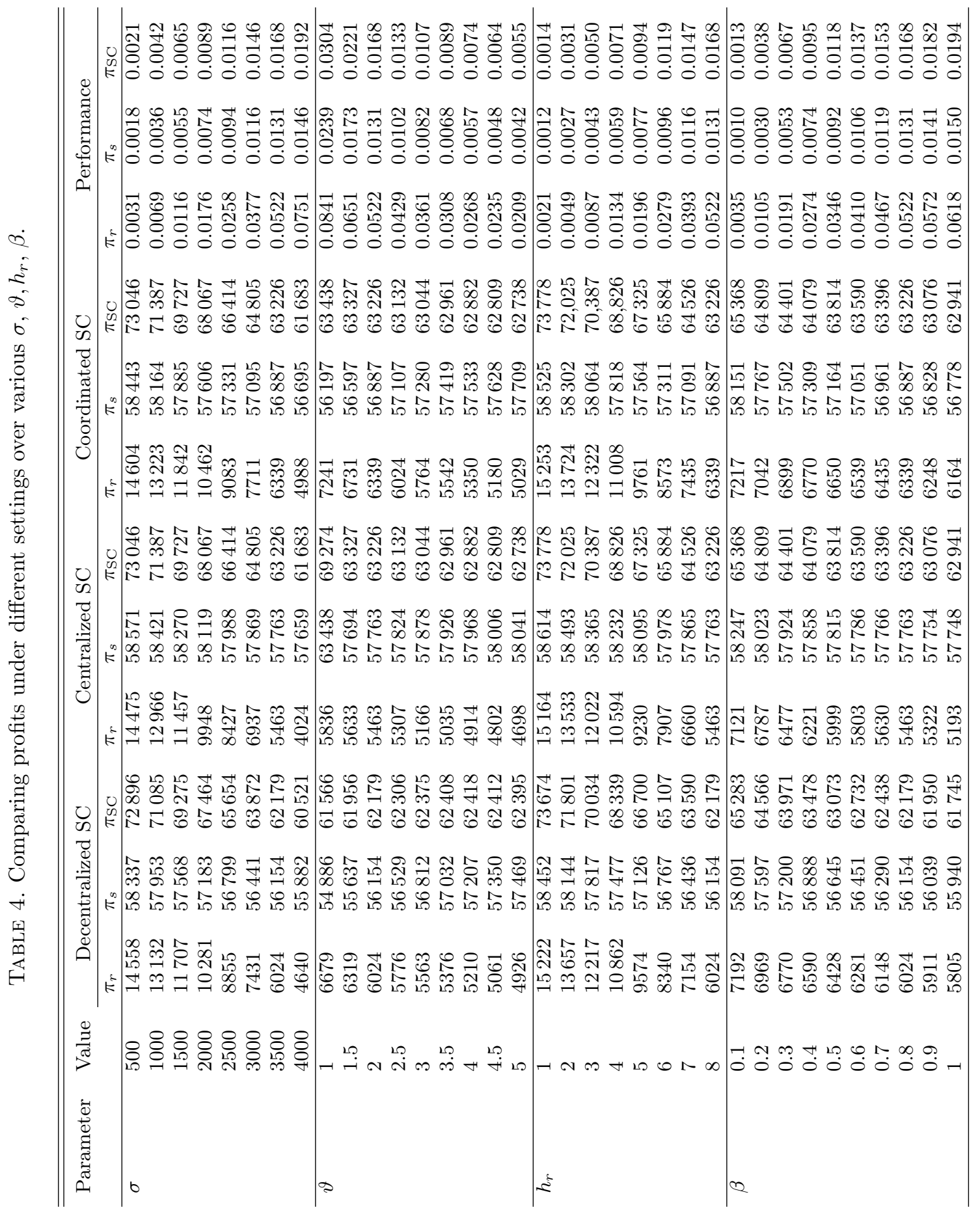




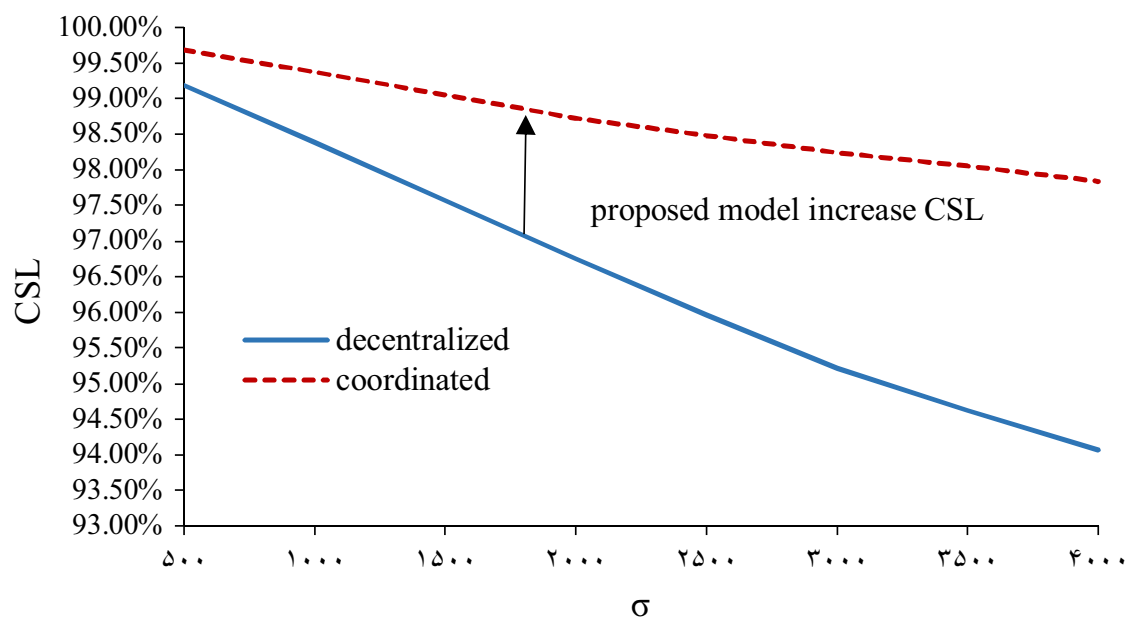

FiguRE 2. CSL vs. $\sigma$ under different models.

Table 3 compares the decision variables alongside CSL and $d$ in the decentralized and coordinated settings by changing $\sigma, \vartheta, h_{r}$, and $\beta$. As a remark, in Table 3, data of parameters are considered based on Test problem 5. From Table 3, as the uncertainty $\sigma$ increases, $T$ decreases in the decentralized and coordinated settings, and $n$ grows. Moreover, as uncertainty grows, CSL reduces in the decentralized and coordinated settings albeit the CSL reduction under the coordination scheme is considerably less. This suggests that the proposed discount contract is important when an SC faces high demand uncertainty. Likewise, by increasing the shortage cost $\vartheta$, retailer's holding cost $h_{r}$, and the lost sale demand $\beta$, a coordinated plan can enhance the CSL relative to the decentralization.

Table 4 compares profits of entire SC and its participants in three settings by changing $\sigma, \vartheta, h_{r}$, and $\beta$. As a remark, in Table 4, data of parameters are considered based on Test problem 5. As $\sigma$ increases in the decentralization, the retailer's profit drops from 14558.27 to 4639.51 while the supplier's profit drops from 58337.36 to 55881.53 . By applying the coordination scheme, the percent change in the retailer's profit increases from 0.0031 to 0.0751 , and the percent change in the supplier's profit increases from 0.0018 to 0.0146 . This suggests that the proposed discount contract benefits a highly unpredictable SC.

Next, as $h_{r}$ increases, profits of SC and its participants decrease under all structures. The retailer's profit suffers more under the centralization. However, the discount contract improves whole SC profit all members' profit, especially the retailer.

Further, as $\beta$ grows, both SC actors' profit increase under the proposed quantity discount contract compared to the decentralization. Therefore, the developed quantity discount contract is useful when SC faces lost sale.

As a remark, in this study, conducting sensitivity analysis by comparing decisions under decentralization and centralization decision models helped us to provide supply chain managers with interesting decision models.

Figures 2 and 3 depict the changes in CSL for various values of demand uncertainty, $\sigma$ and lost sale, $\beta$. Figure 2 illustrates the changes in the CSL under decentralized and coordinated settings by increasing $\sigma$. As $\sigma$ increases, CSL reduces in both settings. However, the decrease in the CSL in the coordination is less than that of the decentralization. Thus, the quantity discount scheme is of great practical application to improve customer satisfaction (offering a higher service level) when a SC faces high demand uncertainty. This result has not been discussed in the previous studies conducted in the periodic review inventory $(e . g .[26,40])$.

Figure 3 analyzes CSL under different structures as $\beta$ changes. According to Figure 3 , as $\beta$ increases, the proposed quantity contract improves the CSL faster relative to the decentralization. Thus, the discount contract is of high significance when lost sale rate is high. This finding demonstrates the importance of CSL for SC 


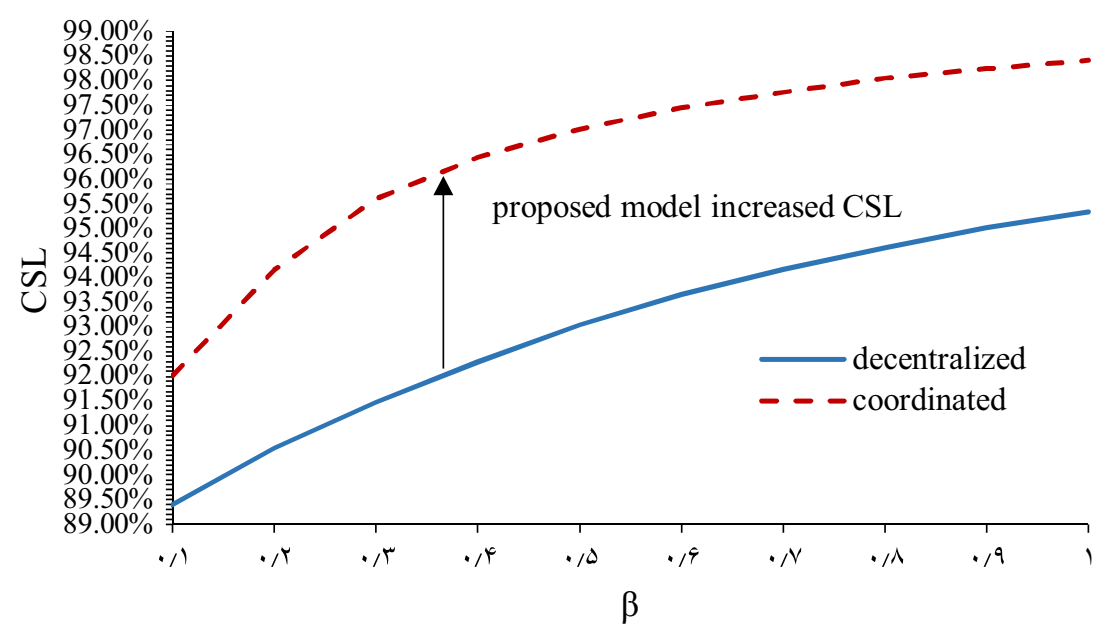

FigURE 3. CSL vs. $\beta$ under the decentralized and coordinated models.

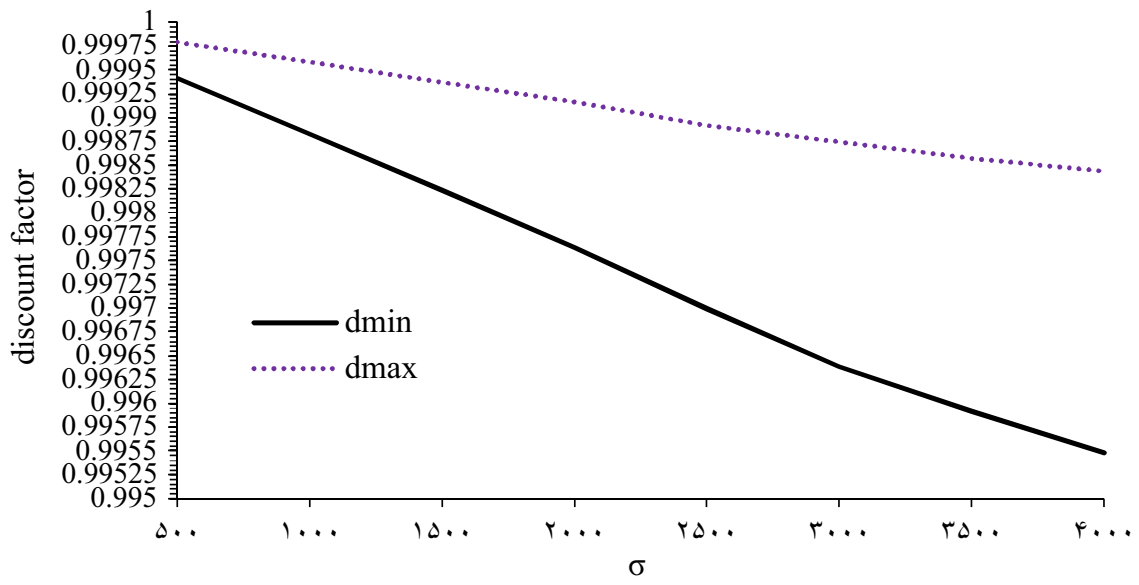

FiguRE 4. Values of $d_{\min }$ and $d_{\max }$ vs. $\sigma$.

managers and the number of stock-outs while coordinating the SCs, especially where the inventory systems might face shortages. This result helps SC managers better provide satisfied service levels. However, in the studies of Johari et al. [26] and Nouri et al. [40], the effect of CSL on the inventory system is ignored.

Figure 4 illustrates the changes in $d_{\min }$ and $d_{\max }$ by increasing demand uncertainty, $\sigma$. As $\sigma$ increases, the difference between $d_{\min }$ and $d_{\max }$ grows, suggesting that the proposed coordination model appeals to both SC members. As a result, the proposed quantity discount contract acts quite well even under high level of demand uncertainty. This finding is in contrast to the studies of Johari et al. [26] and Nouri et al. [40] which concluded that as demand uncertainty increases, $d_{\min }$ comes closer to $d_{\max }$. The proposed contract would be more attractive for the retailer as he could take the chance of receiving more discount from supplier.

Figures 5-7 compare the SC profit and both SC members' profit under various values of demand uncertainty, $\sigma$, shortage cost, $\vartheta$, and retailer holding cost, $h_{r}$, respectively. Figure 5 shows the changes in profit for each member and whole SC by increasing demand uncertainty, $\sigma$. As $\sigma$ grows, the performance of the overall SC 


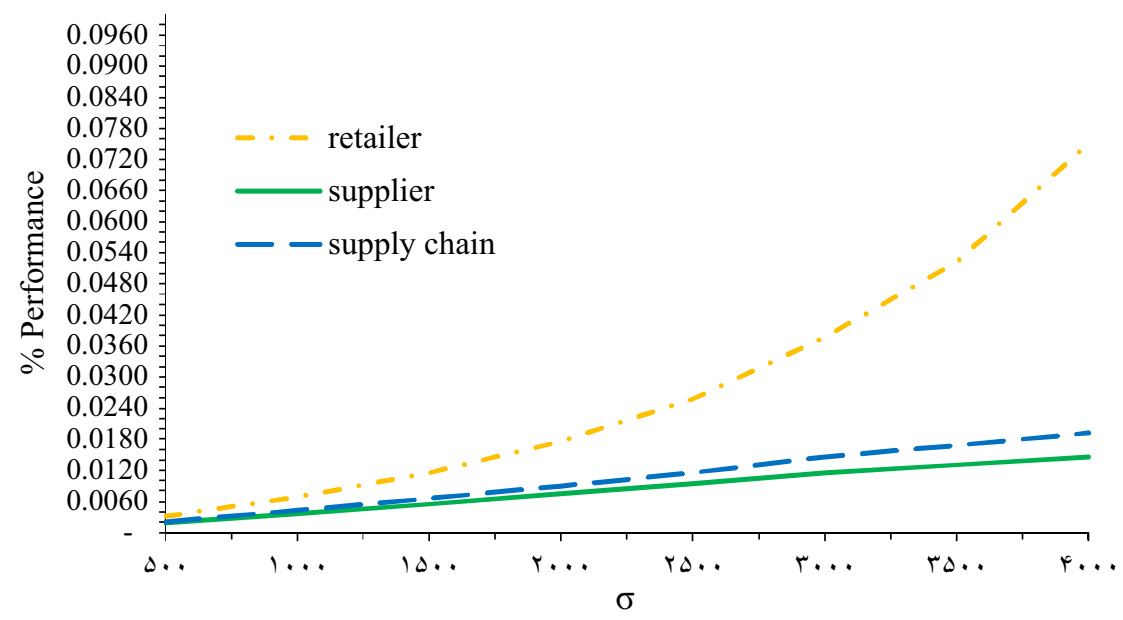

FIGURE 5. SC profit and retailer's and supplier's profit vs. $\sigma$.

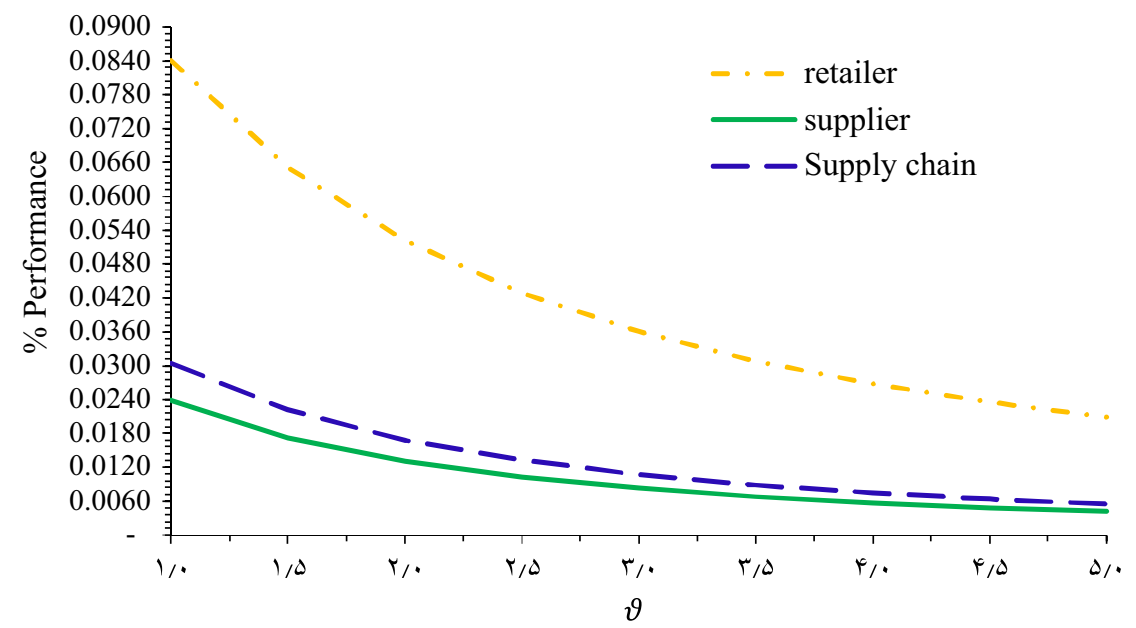

FigURE 6. Analysis of profit improvement vs. $\vartheta$.

and each SC actor improve under the coordination compared to the decentralization. The result shows that the quantity discount contract is of high benefit to the SC members when the SC faces high demand uncertainty.

As shown in Figure 6, as the shortage cost, $\vartheta$ grows, the performance of the SC and both members decrease. Indeed, the shortage cost can diminish SC performance under decentralization. However, a coordination plan can make better profitability.

Figure 7 illustrates the performance of each SC member and the entire SC with respect to the retailer's holding cost, $h_{r}$. As $h_{r}$ grows, profits of two participants enhance in the coordinated model and the difference in the profits of the supplier and retailer increases. Thus, under a high retailer holding cost, the proposed coordination policy is desirable for all members. This result is in contrary to the study of Johari et al. [26] which concluded that the whole SC performance reduces by increasing the retailer's holding cost. This discrepancy is due to the backordered shortages considered in their model. When the shortages are backordered, the retailer's 


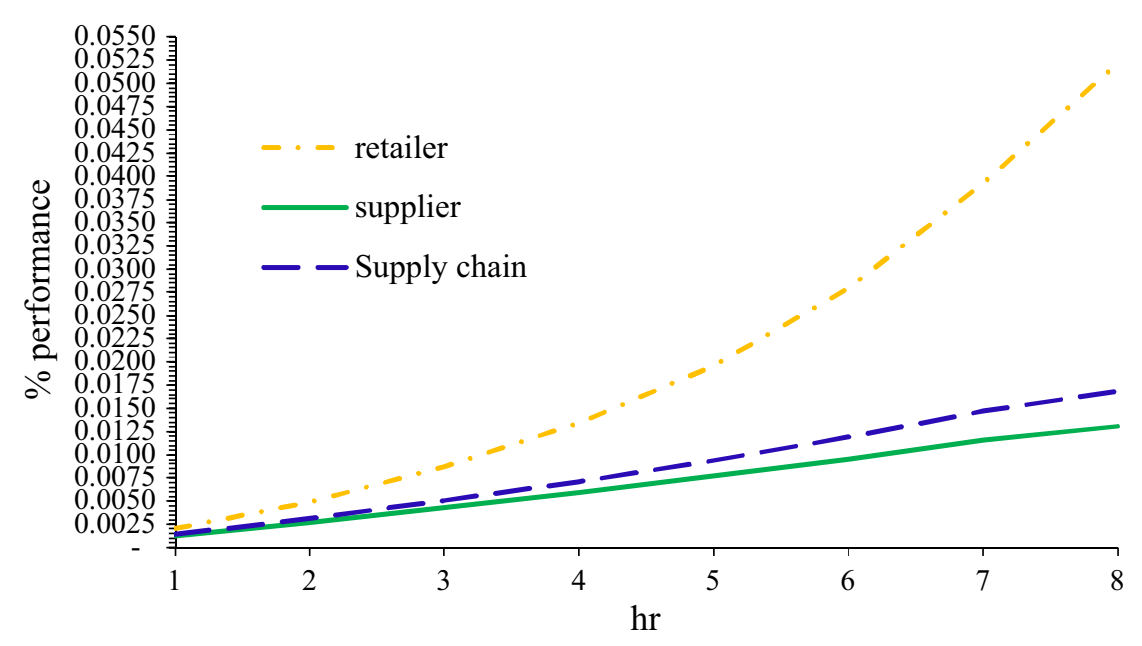

FiguRE 7. Comparison of profit for each member vs. retailer holding cost.

shortage will not impact on the manufacturer's profit and thus it will decrease the channel performance as the retailer's holding cost increases.

\subsection{Case study}

DP is a leading pharmaceutical distribution company. Due to confidentiality reasons, an artificial name is utilized for the distribution company. The mission of DP is to procure human medicine, animals and birds medicines, and medical equipment to distribute and sell both medicine and equipment throughout Iran. The government directly monitors performance and service level of pharmaceutical distribution companies to keep health and safety of society and to prevent medicine stock-out, especially in remote and deprived areas of country. The high ordering cost in remote and deprived areas, which is due to the long distance of these areas from pharmaceutical distribution companies, is one of the primary issues in pharmaceutical industry in Iran. In Iran, pharmaceutical ordering system is based on visiting mechanism due to the large number of medicines. Meanwhile, the pharmaceutical distribution company should firstly send its visitors to receive pharmaceutical orders from demand points (i.e. pharmacies and hospitals). Then, after taking lead time, the pharmaceutical orders are delivered to the demand points through trucks. Hence, in Iran pharmaceutical industry, the ordering cost includes transportation cost of vehicles and costs of inhabitancy and travel of visitors of pharmaceutical distribution companies. DP using modern knowledge and technology under government regulations aims to service all patients and customers with high availability and accessibility, especially in remote and deprived areas. In doing so, due to vastness of Iran country and various demand points, DP established eighteen sub distribution centers in different provinces to properly satisfy pharmaceutical needs. One of these distribution centers is located in Zahedan province.

We focus on Zahedan province as a remote and deprived area and investigate distribution of Insulin medicine. Hospitals and pharmacies of Zahedan province face stochastic demand of Insulin. Due to the long distance $(1485 \mathrm{~km})$ between Zahedan (e.g. demand point) and central distribution center of DP in Tehran, a large warehouse is established in the center of Zahedan city under Food and Drug Administration. The warehouse constructed in Zahedan is regarded as the pharma-supplier of pharmaceutical supply chain (PSC). On the other hand, Nikshahr city is selected as a point of Insulin demand. It is located in the southwest of the Zahedan province, $515 \mathrm{~km}$ from Zahedan capital. Insulin demand is with mean $D=22000 \mathrm{Insulin} /$ year and standard deviation of $\sigma=8000$. We treat hospitals and pharmacies of Nikshahr as one pharma-retailer in the investigated PSC because distances among them is negligible. Due to the negative effects of Insulin shortage on the patients' 


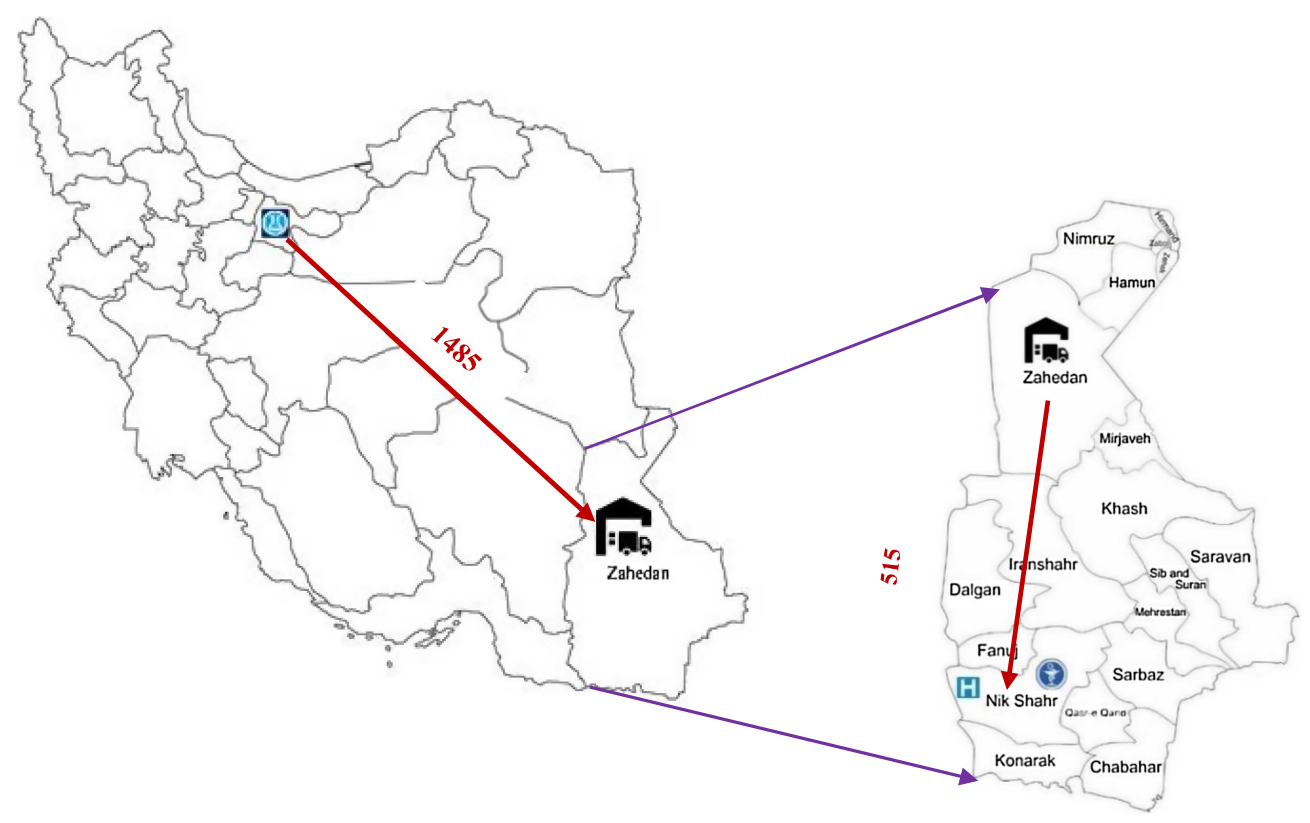

FiguRE 8. Framework of Insulin distribution from DP to Zahedan province.

health, the pharma-supplier is not permitted to face with Insulin shortage according to the government regulations. In addition, as mentioned in the study of Chopra and Meindl [11], the stock-out for the products with the high-level requirement of availability (i.e. Insulin) is not allowed. Therefore, the pharma-supplier should keep proper stock of Insulin medicines under demand uncertainty with unit holding cost of $h_{s}=\$ 2 /$ Insulin per year.

In the pharmaceutical SC in Iran, the periodic review replenishment policy $(T, R)$ is the most common and well-established inventory system due to the large number of medicines. Thus, the pharma-retailer adopts the periodic review inventory system to satisfy the customers' needs. Due to the long distance between pharmasupplier (Zahedan city) and pharma-retailer (Nikshahr city), the pharma-retailer incurs the high cost of vehicle transportation and visitors' inhabitancy and travel from pharma-supplier site for each order placed. Hence, the high ordering cost of $A_{r}=\$ 130$ is spent by the pharma-retailer for each order placed. The pharma-supplier gets the pharmaceutical orders of pharma-retailer in fixed periods, regarding the review periods of pharmaretailer $(T)$. Meanwhile, the pharma-supplier's lot size (orders from DP) is an integer multiple of the orders of pharma-retailer $(n D T)$ (which is well addressed in the prior works (e.g. [8]). Moreover, due to the long distance between the DP (Tehran city) and pharma-supplier (Zahedan city), the pharma-supplier incurs the high cost of vehicle transportation and visitors' inhabitancy and travel from DP for each order placed. Therefore, the pharma-supplier spends high ordering cost with $A_{s}=\$ 120$ for each order. In addition, the pharma-supplier pays cost of $e_{s}=\$ 29 /$ Insulin for purchasing Insulin medicines from DP. The placed orders of the pharma-retailer are received after $L=5$ days because of the lead time of pharma-supplier. The pharma-retailer purchases Insulin medicines from pharma-supplier with cost $w=\$ 34 /$ Insulin, and then he sales them to the patients and customers with price $p=\$ 36 /$ Insulin. Moreover, the pharma-retailer should hold Insulin stock with cost $h_{r}=\$ 4 /$ Insulin for each stock to provide high CSL. The pharma-retailer faces Insulin stock-out with rate of lost sale $\beta=0.7$ and he should spend cost $\vartheta=\$ 0.8 /$ Insulin for each Insulin shortage. In addition, due to the dominant power of pharma-retailers in Iran pharmaceutical industry, the pharma-retailer's bargaining power is defined as $\alpha=0.9$ against the pharma-supplier which is $(1-\alpha)=0.1$.

Figure 8 illustrates the framework of Insulin distribution from DP (as an external supplier of the pharmaceutical SC), Zahedan (as a warehouse of the pharma-supplier), and hospitals and pharmacies of Nikshahr (as the 
TABLE 5. Average demand of hospitals and pharmacies of Nikshahr.

\begin{tabular}{lll}
\hline \hline Customer & Location & $\begin{array}{l}\text { Mean demand } \\
\text { of Insulin/year } \\
\text { (Boxes) }\end{array}$ \\
\hline H Nikshahr Hospital & Nikshahr & 4200 \\
H 22 Bahman Hospital & NikShahr & 7500 \\
- Arya pharmacy & Nikshahr & 2100 \\
- Sobhan pharmacy & Nikshahr & 1900 \\
- Nikshahr pharmacy & Nikshahr & 1700 \\
- Farabi pharmacy & Nikshahr & 2800 \\
- Hasani pharmacy & Nikshahr & 1800 \\
\hline
\end{tabular}

TABLE 6. Comparing decision-making structures under PSC.

\begin{tabular}{lcccccccccc}
\hline \hline $\begin{array}{l}\text { Decision- } \\
\text { making } \\
\text { structure }\end{array}$ & $T$ (day) & $k$ & $n$ & CSL & $\begin{array}{l}\text { Pharma- } \\
\text { retailer } \\
\text { profit }(\$)\end{array}$ & $\begin{array}{l}\text { Pharma- } \\
\text { supplier } \\
\text { profit }(\$)\end{array}$ & $\begin{array}{l}\text { Whole } \\
\text { SC } \\
\text { profit }(\$)\end{array}$ & $d_{\text {min }}$ & $d_{\text {max }}$ & $d_{\text {shared }}$ \\
\hline $\begin{array}{l}\text { Decentralized } \\
\text { model }\end{array}$ & 12.92 & 1.54 & 2 & $93.93 \%$ & 24772.45 & 102889.61 & $127662.06-$ & - & - \\
$\begin{array}{l}\text { Centralized } \\
\text { model }\end{array}$ & 12.47 & 1.98 & 2 & $97.93 \%$ & 23763.29 & 105910.61 & $129673.91-$ & - & - \\
$\begin{array}{l}\text { Coordinated } \\
\text { model }\end{array}$ & 12.47 & 1.98 & 2 & $97.93 \%$ & 26583.12 & 103090.79 & 129673.91 & 0.9959 & 0.9986 & 0.9961 \\
\hline
\end{tabular}

pharma-retailer). In addition, Table 5 indicates the average demand of hospitals and pharmacies of Nikshahr as the pharma-retailer.

Table 6 indicates the results of analyzing different settings according to the data of the investigated case study. From Table 6 , the pharma-retailer obtains $\$ 24772.45$ benefit by optimizing its own profit individually under the decentralized setting. While, the pharma-retailer's profit under the centralization decreases to $\$ 23763.29$. Meanwhile, the pharma-retailer by changing its local decisions to global decisions incurs losses $\$ 23763.29-\$ 24772.45=\$-1009$.16. However, the centralization improves the whole SC profit from $\$ 127662.06$ to $\$ 129673.91$ as well as the profit of pharma-supplier from $\$ 102889.61$ to $\$ 105910.61$. Thus, the centralization is not desirable for the pharma-retailer. In such a setting, a coordinated plan can be designed to offset the losses of the pharma-retailer.

Thus, in the investigated PSC, we develop quantity discount contract as a coordination scheme to encourage both pharma-retailer and pharma-supplier to make decisions similar to the centralized setting. Under the developed quantity discount scheme, the pharma-supplier aims to decrease the wholesale price to incentivize the pharma-retailer to alter its decentralized choices to the centralized one. As illustrated in Table 6, the quantity discount contract reveals that by decreasing wholesale price $(d w=\$ 33.87)$ pharma-retailer achieves more profit (i.e. $\$ 26583.12$ ) compared to the decentralization (i.e. $\$ 24772.45$ ). Moreover, the pharma-supplier obtains $\$ 103090.79$ profit in the quantity discount contract compared to the decentralization (i.e. $\$ 102889.61$ profit). In addition, the whole PSC profit improves under the proposed coordinated setting relative to the decentralized one (\$129673.91 vs. \$127662.06). Thus, running SC coordination is of high importance in the investigated PSC regarding critical and strategic role of pharmaceutical in health of society, complexity of pharmaceutical inventory management, and demand uncertainty. 


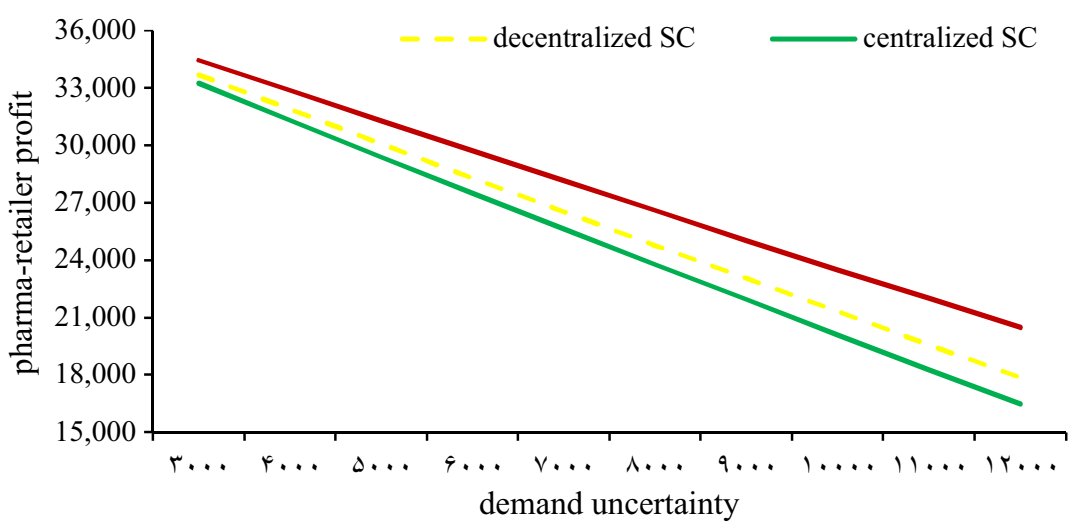

FiguRE 9. Pharma-retailer profit over $\sigma$ under three decision-making structures.

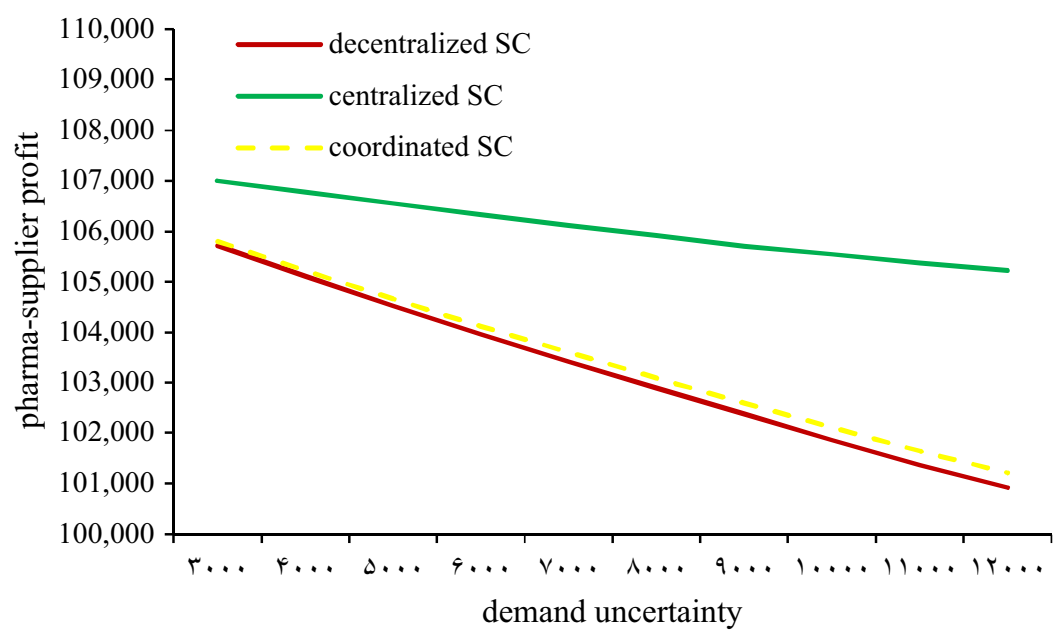

FiguRE 10. Pharma-supplier profit over $\sigma$ under three decision-making structures.

As a remark, considering a real case data, based on the modification of key parameters (inventory holding cost, shortage cost, lost sale rate, and demand uncertainty) some figures (Figs. 8-15) are carried out to provide sensitivity analysis.

\section{Insight 1. Profits of both PSC members and whole PSC change over variation of the demand uncertainty.}

As a remark, Figures 9 and 10 indicate how the profit of supply chain actors (i.e. pharma-retailer and pharmasupplier) is changed by increasing demand uncertainty, $\sigma$, and Figure 11 illustrates the changes in the profit of whole supply chain over growing $\sigma$ under different structures.

From Figures 9 and 10, as $\sigma$ increases, the supply chain actors' profit reduces under all structures. However, in the discount contract, all actors' profit improves in comparison with the decentralized one. Moreover, for greater values of $\sigma$, the proposed discount contract is able to bring more profits for both actors compared to the decentralization. Therefore, when SCs face high demand uncertainty, adopting quantity discount contract will be appealing for SC actors as it leads to great benefit for all SC members. This finding reflects the application of the coordination scheme when SCs face high demand uncertainty. 


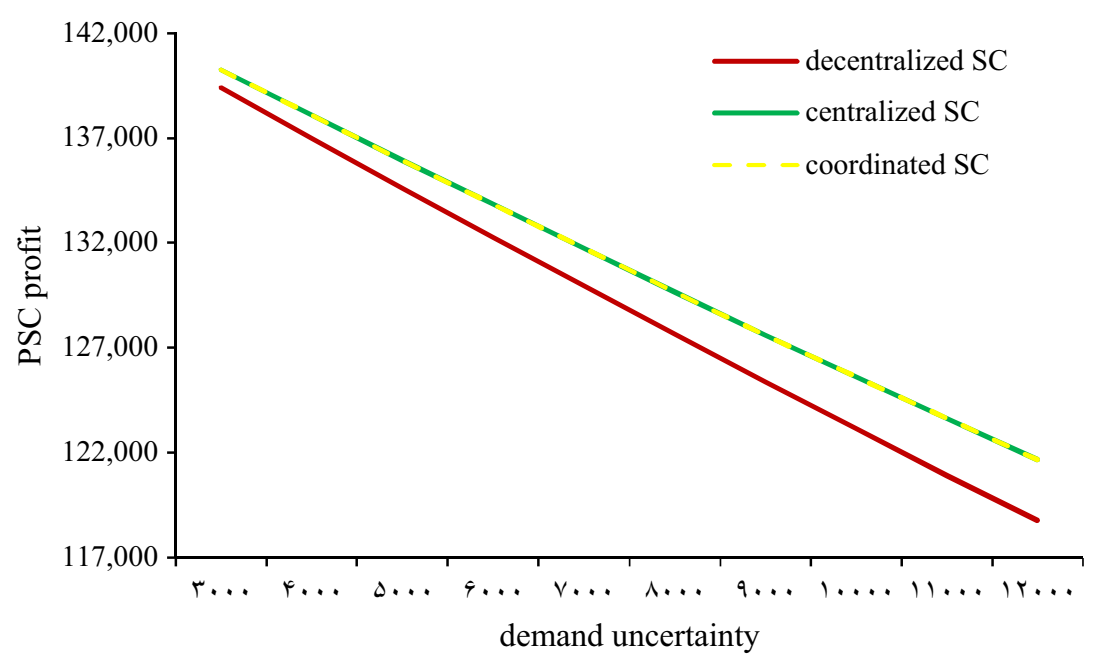

FIGURE 11. PSC profit over $\sigma$ under various models.

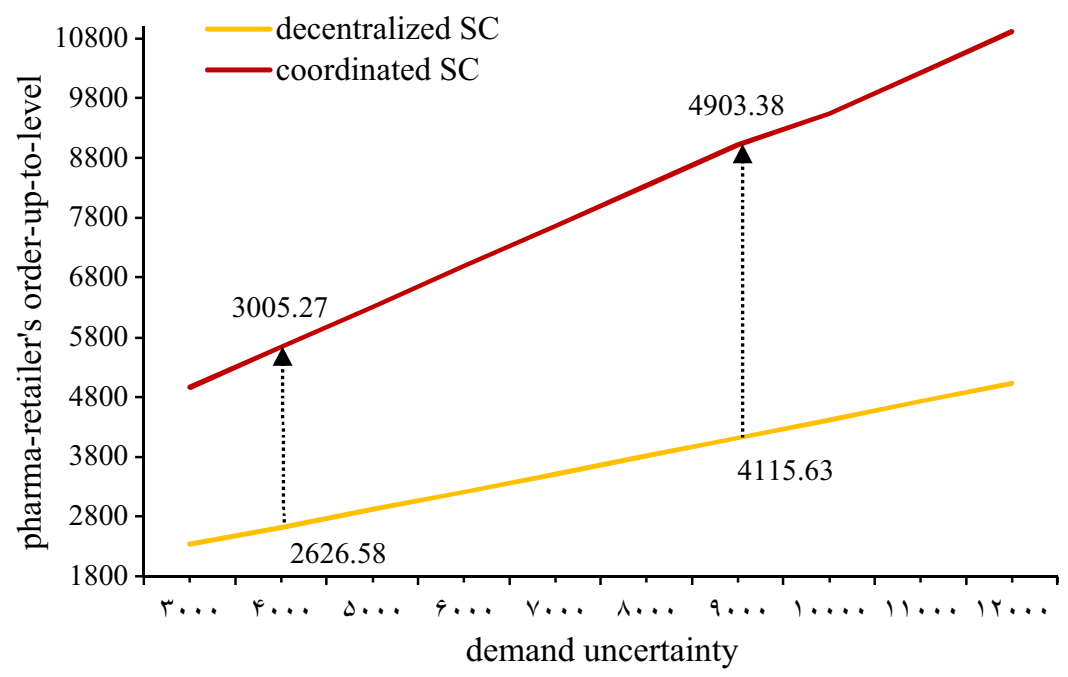

FiguRE 12. Order-up-to-level over $\sigma$ under decentralized and coordination models.

According to Figure 11, rising $\sigma$ decreases the PSC profit under all models. However, the results reveal the effectiveness of the developed discount scheme to improve the PSC profit compared to the decentralization under various amounts of demand uncertainty. In addition, from Figure 11, the proposed discount scheme is able to bring channel coordination for the considered PSC as it creates profit for PSC equal to that of centralized setting. Hence, under demand uncertainty, using the discount contract is of high applicability as it is able to create satisfactory solution for whole SC and boost all SC actors' profit.

\section{Insight 2. Order-up-to-level is influenced by demand uncertainty.}

Figure 12 illustrates the changes in the pharma-retailer's order-up-to-level by increasing demand uncertainty, $\sigma$. From Figure 12, as $\sigma$ increases, the pharma-retailer should increase its order-up-to-level under both decentralized and coordinated settings to decrease shortages. Moreover, by increasing $\sigma$, the difference between 


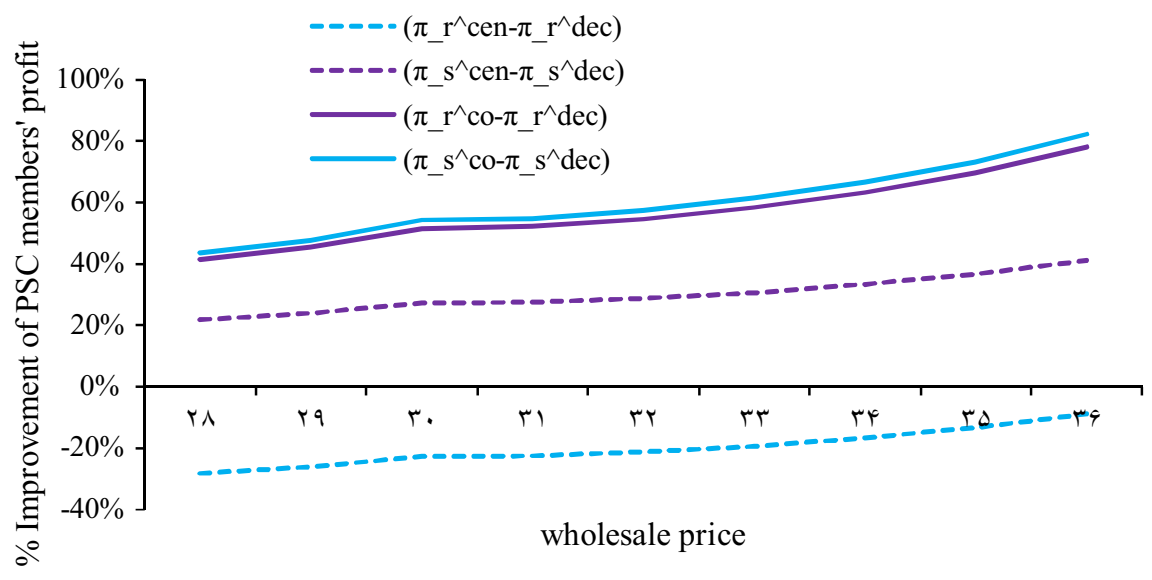

FIGURE 13. PSC members' profit improvement over $w$ under centralized and coordination models.

order-up-to-level under the decentralized and coordinated settings increases. For small amounts of $\sigma$ (i.e. 4000) the order-up-to-level increases about $14.42 \%$ under the discount scheme. While, the increase in order-up-to-level for greater values of $\sigma$ (i.e. 9000) is about $19.14 \%$ which incurs more costs for the pharma-retailer. Therefore, from the high-level requirement of availability point of view, the pharma-retailer should make tradeoff between its imposed costs under each decision-making structure. Meanwhile, in such a case, applying the proposed discount scheme could be of challenging issue. Thus, the proper implementation of quantity discount scheme is of high importance to help SC managers to have a better understanding when their SCs face high demand uncertainty and to overcome this issue. Although the studies of Johari et al. [26] and Nouri et al. [40] investigate periodic review inventory system under uncertain demand, they do not analyze the impact of demand uncertainty on the order-up-to-level provided by the retailer.

\section{Insight 3. Changes in the PSC members' profit over variation of the wholesale price.}

Figure 13 illustrates trend of profit improvement for the pharma-supplier and pharma-retailer in the centralization relative to the decentralization $\left(\pi_{i}^{\text {cen }}-\pi_{i}^{\text {dec }}\right)$ and coordination relative to the decentralization $\left(\pi_{i}^{\text {co }}-\pi_{i}^{\text {dec }}\right)$ by rising wholesale price, $w$. According to Figure 13, the profit of both actors boosts by increasing $w$ under both centralization and coordination relative to the decentralization. However, the centralization leads to more difference between profitability of actors. The results demonstrate that the quantity discount contract significantly boosts all PSC members' profit and decreases the difference between the profit of them as $w$ rises. Meanwhile, conducting the coordination scheme can remarkably reach the profit improvement of pharma-retailer up to pharma-supplier's profit improvement through compensating the incurred losses of pharma-retailer in the centralization. Therefore, the coordination scheme can boost incentive for the pharma-retailer to contribute in the coordination in addition to inspire the pharma-supplier to accept the coordination through bringing more benefit for all PSC members in comparison with the decentralization. Moreover, for greater amounts of $w$, implementing the quantity discount contract leads to more profitability for all PSC actors in comparison with the centralization and decentralization. As a result, for SC managers, increasing wholesale price concerns with decision-making structure such that the proposed quantity discount contract as a coordination structure can remarkably improve the profit of retailer and supplier even when wholesale price increases. This improvement is not reached under the decentralized and centralized structures.

\section{Insight 4. CSL is affected by pharma-retailer's holding cost.}

Figure 14 depicts the changes in CSL by increasing pharma-retailer holding cost, $h_{r}$, under decentralized and coordinated structures. From Figure 14, by increasing $h_{r}$, CSL decreases under all settings. However, decrease in CSL under the coordinated scheme is remarkably less than the decentralization as CSL in the coordinated 


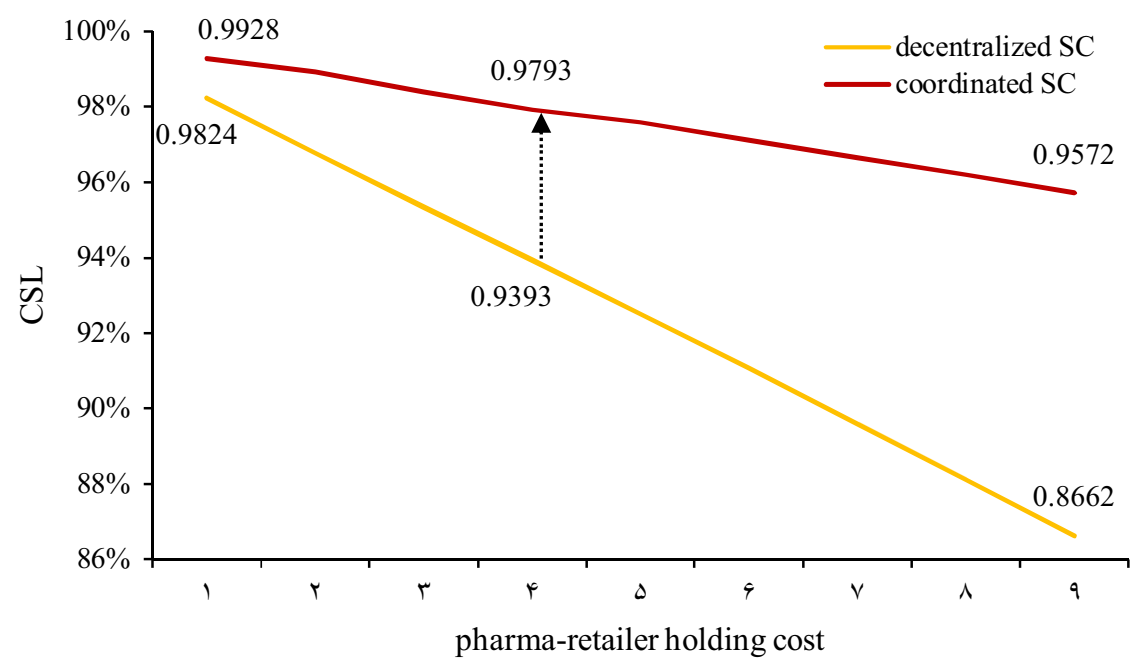

FIGURE 14. Changing CSL over $h_{r}$ under decentralized and coordination models.

model decreases from $99.28 \%$ to $95.72 \%$ while CSL in the decentralization decreases from $98.24 \%$ to $86.62 \%$. The results reveal that in case of high-level requirement of service level and high retailer's holding cost (which is consistent with the investigated PSC), applying the quantity discount contract is more robust than the decentralized setting as it maintains the satisfaction level of customer service even when retailer's holding cost is high. As a result, for SC managers, increasing CSL relates to holding cost of retailer such that any increase in the holding cost will diminish CSL. Therefore, maintaining customer service in the satisfied level requires an incentive coordination scheme as demonstrated in Figure 14. Accordingly, to coordinate inventory systems, it is important to take the retailer's holding cost into account, which can negatively affect the CSL and whole SC performance. However, in the studies of Johari et al. [26] and Nouri et al. [40], the impact of retailer's holding cost on the CSL is not analyzed.

\section{Insight 5. Profits of both PSC members change under the variation of pharma-retailer holding cost.}

Figure 15 indicates trend of profit improvement for the pharma-retailer and pharma-supplier in the centralized setting relative to the decentralized $\left(\pi_{i}^{\mathrm{cen}}-\pi_{i}^{\mathrm{dec}}\right)$, and coordinated plan relative to the decentralized model $\left(\pi_{i}^{\text {co }}-\pi_{i}^{\text {dec }}\right)$ by increasing pharma-retailer's holding cost, $h_{r}$. According to Figure 15 , the pharma-retailer's profit decreases by increasing $h_{r}$ under the centralized structure relative to the decentralized structure. However, rising $h_{r}$ improves considerably the profit of pharma-supplier under the integrated setting relative to the decentralized setting. Meanwhile, the pharma-retailer endures losses while the pharma-supplier benefits by increasing $h_{r}$ under the centralized structure compared to the decentralized one. For greater values of $h_{r}$, the difference between pharma-supplier's profitability and pharma-retailer's losses increases under the centralization. Therefore, the pharma-retailer does not agree on the centralization. Conversely, the quantity discount contract can boost both PSC members' profit relative to the decentralized structure by increasing $h_{r}$, especially the pharmaretailer's profit. Accordingly, the quantity discount can properly convince the pharma-retailer to take part in the coordinated plan. Thus, under high retailer's holding cost, running quantity discount scheme as adopted in the PSC, is of high implication as it is capable of bringing more profits for all SC participants relative to the decentralization if properly applied. 


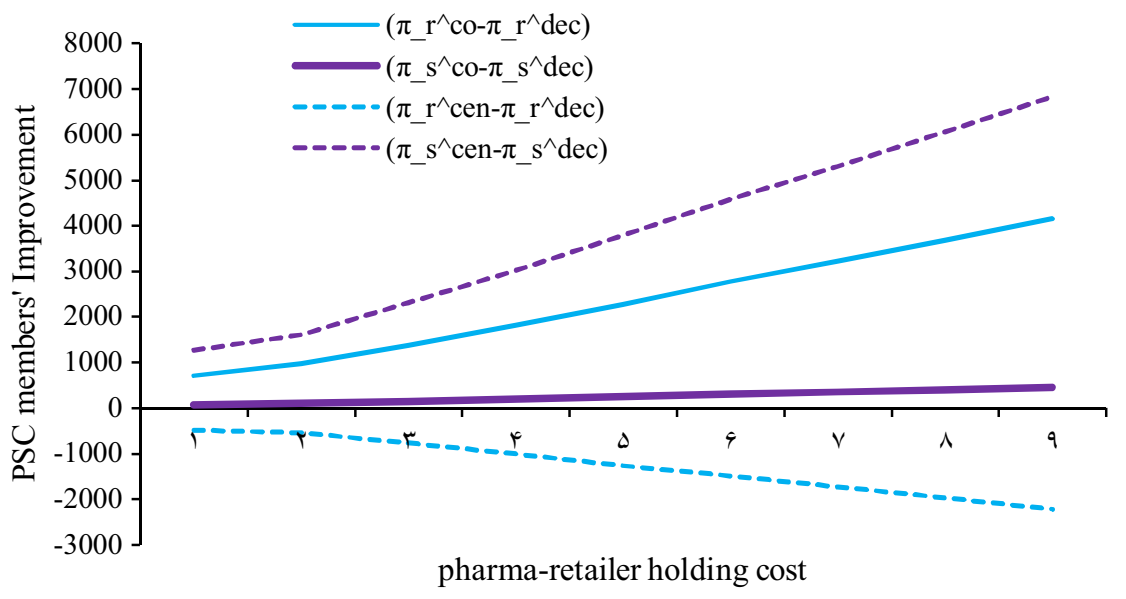

FIGURE 15. PSC members' profit over $h_{r}$ under centralized and coordination models.

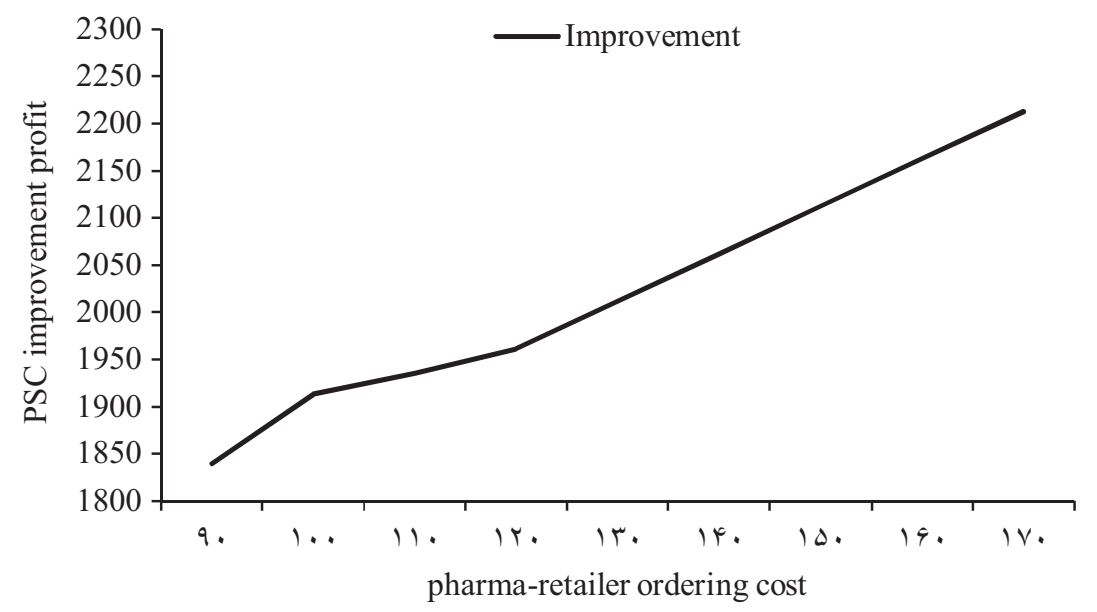

FiguRE 16. PSC profit over $A_{r}$ under coordination model.

\section{Insight 6. PSC profit changes under the variation of pharma-retailer's ordering cost.}

Figure 16 depicts trend of improvement in the profit of whole PSC under the coordinated setting relative to the decentralized one $\left(\pi_{\mathrm{SC}}^{\mathrm{co}}-\pi_{\mathrm{SC}}^{\mathrm{dec}}\right)$ by increasing the pharma-retailer's ordering cost, $A_{r}$. As mentioned before in Iran pharmaceutical industry, high ordering cost is of challenging issue. From Figure 16, the whole PSC profit boosts significantly under the coordination scheme compared to the decentralization by increasing $A_{r}$. This result reveals the application of the quantity discount contract as PSC benefits even when retailer's ordering cost is high.

\section{Insight 7. CSL changes over the pharma-retailer's shortage cost.}

Figure 17 illustrates the changes in CSL by increasing pharma-retailer's shortage cost, $\vartheta$ under the decentralized and coordinated structures. From Figure 17, CSL increases under all structures by increasing $\vartheta$ while the proposed coordinated scheme improves CSL more than the decentralized one. Under increase of pharmaretailer's shortage cost, CSL in the proposed coordination scheme increases smoothly (97.74\%-98.70\%), but that of the decentralized enhances rapidly $(92.21 \%-97.74 \%)$. Meanwhile, when retailers face low shortage cost, 


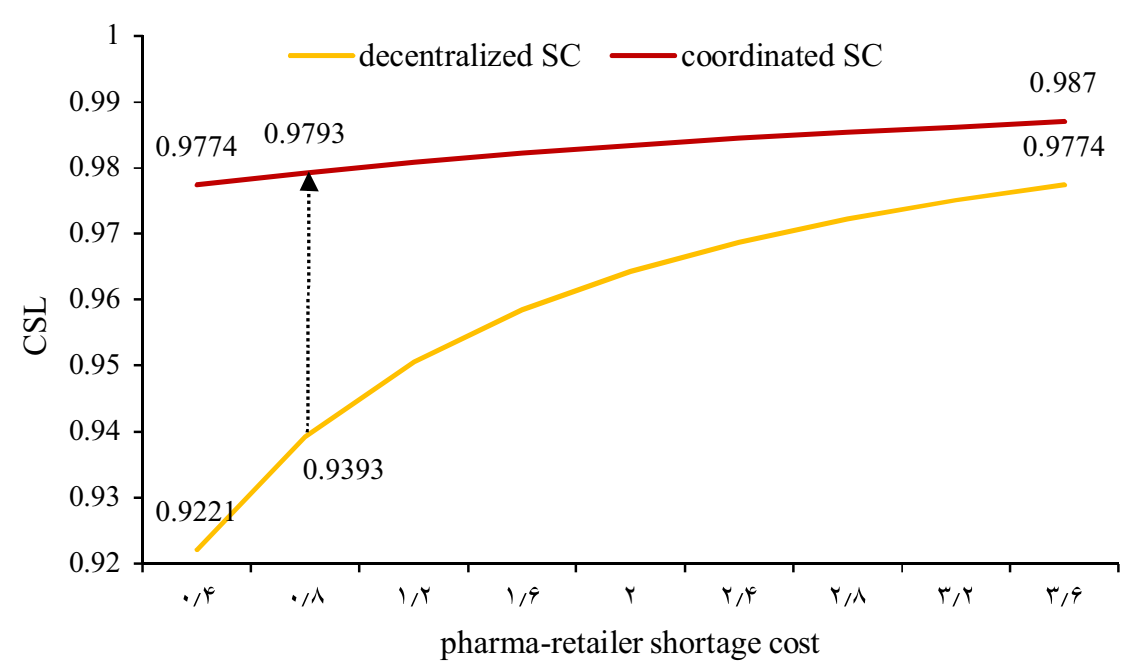

Figure 17. Changing CSL over $\vartheta$ under decentralized and coordination models.

they provide small service level to the customers. Thus, under such a situation (low shortage cost), using the proposed quantity discount contract as adopted in the PSC, is of high applicability as it improves CSL considerably compared to the decentralized setting. While under high shortage cost, the retailers set high CSL and then the difference between the CSL under the coordination and decentralization decreases as indicated in Figure 17. Moreover, by increasing $\vartheta$, the difference between CSL under the decentralized and coordinated models decrease. From Figure 17, for high value of shortage cost (i.e. 3.6), the decentralized setting can achieve CSL up to $97.74 \%$, which is resulted by the coordination scheme in the small value of shortage cost. The shortage cost of the investigated PSC relates to the beginning trend of Figure 17 (i.e. 0.8) as the difference between CSL under the decentralized and coordination is remarkably high. As a result, running the proposed quantity discount contract is of great applicability in the PSC as it increases CSL from $93.93 \%$ to $97.93 \%$ compared to the decentralized structure. This analysis has not been previously conducted in the context of periodic review ordering model $($ e.g. $[26,40])$. In addition, previous works in this research area have not analyzed the impact of retailer's shortage cost on the provided service level in the periodic review replenishment setting.

\section{Conclusion}

In health sector, improving customer service level (CSL) for pharmaceuticals in the presence of demand uncertainty is of high importance. In practice, the inventory decisions made by pharmacies directly affect the CSL. In the current study, we analyze how coordinating inventory decisions of pharmacies and suppliers can benefit society by improving CSL and SC members' profits. Motivated by a case study, a supplier-retailer supply chain is examined where the retailer utilizes a periodic review $(T, R)$ ordering system and specifies the order-up-to-level $R$ and review period $T$ simultaneously, while the supplier finds the replenishment multiplier $\mathrm{n}$ for ordering the items. A coordinated scheme through a quantity discount contract is designed to transition between the decentralized and centralized models with minimum and maximum acceptable discounts for both members. We find that the centralization makes more profit for the overall SC but the it may decrease the profit of the downstream member compared to the decentralization. Thus, it is advantageous to use a coordination scheme to incentivize the downstream actor to agree on the centralization. Our model can provide channel coordination whereby both members benefit. Further, the proposed discount contract can improve CSL and reduce shortages. As a result, a simultaneous coordinating replenishment multiplier, review period, and 
order-up-to-level in a supplier-retailer chain can lift SC profit and achieve better customer satisfaction. The main findings of our model are summarized as follows:

- In a periodic review ordering technique, the retailer's choices on safety stock and review period influence the CSL, which in turn affects the sales volume and profit of the SC participants. In this study, the results suggest that the retailer's periodic review replenishment decisions under the decentralized model cannot optimize the whole SC profitability and reduce the CSL. This issue is likely to cause both profit losses and decreased customer satisfaction, especially in a socially-concerned system, such as pharmaceutical SC. This finding can help SC managers get a clear understanding of this issue.

- The proposed coordination scheme can assist SC managers to enhance the efficiency of the supply chain under the periodic review ordering policy in terms of both service level enhancement and profitability. Using the proposed quantity discount contract, the results show that it is utilizable to induce the retailer to optimize its periodic review replenishment decisions from the whole SC viewpoint and consequently great benefits for all SC participants will be achieved. Moreover, the proposed quantity discount contract significantly improves CSL relative to the decentralized model. For a various number of parameters, the findings indicate that there exists a feasible range for the discount factor which achieves the channel coordination; thereby improving the overall SC profitability and providing a win-win condition for all SC actors.

- The conducted case study and sensitivity analyses can assist managers to better identify the situations in which the lack of coordination on periodic review inventory decisions could considerably mitigate the efficiency of overall SC. The findings suggest that the decentralized model performs poorly under high ordering cost, low shortage cost, and high demand uncertainty. Under such situations, which are more probable in pharmaceutical industry, the proposed coordination model can assist managers to considerably improve the CSL and profitability of the SC participants by coordinating the periodic review replenishment decisions. In real-world situations, there are generally a lot of pharmaceutical items in healthcare systems. Our results help managers identify the items which have priority over others and should be replenished according to the proposed coordination strategy in this article.

This study can be extended in several paths. In this study, the discount contract is used in the periodic review inventory system. However, under other situations, the discount contract has some limitations. For instance, when supply chain faces competitive situations, it will be challenging the use of discount contract as it is simple contract (i.e. it includes one contract parameter). In addition, it will be challenging to use the discount contract when wholesale price is endogenous. Moreover, as competitive situations of the market affect decisions of supply chain managers, investigating coordination contracts for the periodic review inventory under competitive situation is of great importance. As a note for future research, this study can be extended by investigating the periodic review inventory under competitive situation through coordination contracts. In addition, in this study the discount contract is proposed in health sector. However, the coordination contracts can be effectively used in other sectors, such as environment sector, energy sector, and industrial sector. Also, industry sector includes several activities, such as production, inventory, purchasing, and sales and marketing. Such different activities in the industry sector implies that the industry is similar to the supply chain in which management of different decisions is of high significance. Accordingly, this study can be extended for the industry sector by adopting coordination contracts as the complicated situations (i.e. exist of various decisions, such as inventory, production, purchasing, and marketing) need coordination of different decisions. Additionally, this study investigates the level of customer service for the periodic review inventory system by coordinating inventory decisions and accordingly, sensitivity analyses are revolved around key parameters relating to customer service level and inventory system (inventory holding cost, shortage cost, lost sale rate, and demand uncertainty). However, as a note for future research, more research can be conducted to analyze the coordination of supply chains under critical mathematical analyses, such as competitive and disruption situations. Moreover, this article considers that supplier's inventory holding cost is known to the retailer and retailer's inventory holding cost is known to the supplier. However, in many other cases, supplier/manufacturer has private information about its inventory holding cost and it may not be known to the other SC members. Moreover, the retailer does not share its 
information about inventory holding cost with the supplier/ manufacturer. This model can be extended by considering asymmetric information about inventory holding cost.

\section{Appendix A.}

Proof of Proposition 3.1. The second-order derivative of $\pi_{r}(T, k)$ w.r.t. $k$ is $\frac{\partial^{2} \pi_{r}(T, k)}{\partial k^{2}}=-h_{r} \beta \sigma \sqrt{T+L} \varphi(k)-$ $\frac{1}{T}(\vartheta+\beta(p-w)) \sigma \sqrt{T+L} \varphi(k)<0$. Hence, $\pi_{r}(T, k)$ is concave with respect to $k$ for a given $T$.

Proof of Proposition 3.2. The second-order derivative of $\pi_{s}(n)$ w.r.t. $n$ is $\frac{\partial^{2} \pi_{s}(n)}{\partial n^{2}}=-\frac{2 A_{s}}{T n^{3}}<0$. This result proves the concavity of $\pi_{s}(n)$.

Proof of Proposition 3.3. To prove concavity w.r.t. $n$ and $k$ for a given $T$, the Hessian matrix of $\pi_{\mathrm{SC}}(T, k, n)$ w.r.t. $n$ and $k$ is used, which is provided in equation (A.1).

$$
H\left(\pi_{\mathrm{SC}}\right)=\left[\begin{array}{ll}
\frac{\partial^{2} \pi_{\mathrm{SC}}}{\partial n^{2}} & \frac{\partial^{2} \pi_{\mathrm{SC}}}{\partial n \partial k} \\
\frac{\partial^{2} \pi_{\mathrm{SC}}}{\partial k \partial n} & \frac{\partial^{2} \pi_{\mathrm{SC}}}{\partial k^{2}}
\end{array}\right]
$$

where, equation (A.2) calculates the first principal minor, which is always negative.

$$
H_{11}=\frac{\partial^{2} \pi_{\mathrm{SC}}}{\partial n^{2}}=\frac{-2 A_{s}}{n T^{3}}<0 .
$$

Moreover, equation (A.3) indicates the second derivative of SC profit w.r.t. $k$ and $n$. Also, equation (A.4) shows the second derivative of SC profit w.r.t. $k$.

$$
\begin{aligned}
\frac{\partial^{2} \pi_{\mathrm{SC}}}{\partial k \partial n}= & \frac{\partial^{2} \pi_{\mathrm{SC}}}{\partial n \partial k}=\frac{\sigma \sqrt{T+L}(\Phi(k)-1) h_{s} \beta}{2} \\
\frac{\partial^{2} \pi_{\mathrm{SC}}}{\partial k^{2}}= & -\sigma \sqrt{T+L} \varphi(k)\left[h_{r} \beta+\frac{(\vartheta+\beta(p-e))}{T}-\frac{h_{s}(n-1) \beta}{2}\right] \\
H_{22}= & \left(\frac{\partial^{2} \pi_{\mathrm{SC}}}{\partial n^{2}}\right)\left(\frac{\partial^{2} \pi_{\mathrm{SC}}}{\partial k^{2}}\right)-\left(\frac{\partial^{2} \pi_{\mathrm{SC}}}{\partial k \partial n}\right)^{2}=\frac{A_{s} \beta \varphi(k)\left[2 h_{r}-h_{s}(n-1)\right]}{T n^{3}} \\
& +\frac{2 A_{s} \varphi(k)(\vartheta+\beta(p-e))}{T^{2} n^{3}}-\frac{h_{s}^{2} \beta^{2} \sigma \sqrt{T+L}}{4}(\Phi(k)-1)^{2} .
\end{aligned}
$$

Equation (A.5) provides the second principal minor, which is positive under the condition $\frac{A_{s} \beta \varphi(k)\left[2 h_{r}-h_{s}(n-1)\right]}{T n^{3}}+$ $\frac{2 A_{s} \varphi(k)(\vartheta+\beta(p-e))}{T^{2} n^{3}}>\frac{h_{s}^{2} \beta^{2} \sigma \sqrt{T+L}}{4}(\Phi(k)-1)^{2}$. Thus, Hessian matrix is negative definite and $\pi_{\mathrm{SC}}(T, k, n)$ is concave w.r.t. $n$ and $k$.

Proof of Corollary 3.4. In the SC under investigation, under the centralized model, the review period $\left(T^{\mathrm{cen}}\right)$, safety factor $\left(k^{\mathrm{cen}}\right)$, and replenishment multiplier $\left(n^{\mathrm{cen}}\right)$ decisions are determined by optimizing of the overall SC profit function which results in the globally optimal solution for the overall SC. In the decentralization, the review period $\left(T^{\mathrm{dec}}\right)$ and safety factor $\left(k^{\mathrm{dec}}\right)$ decisions are individually optimized according to the retailer's profit and the replenishment multiplier $\left(n^{\mathrm{dec}}\right)$ decision is optimized based on the supplier's profit. However, the retailer's decisions affect the supplier's profit. Thus, the decentralization creates a locally optimal solution for the overall SC as such individual choices are not optimized from the SC standpoint. Accordingly, the overall SC profit under the centralization is greater than that of the decentralization as the SC choices are made so as to maximize the overall SC profit. Mathematically, $\pi_{\mathrm{SC}}^{\text {cen }}\left(T^{\mathrm{cen}}, k^{\mathrm{cen}}, n^{\mathrm{cen}}\right) \geq \pi_{\mathrm{SC}}^{\mathrm{dec}}\left(T^{\mathrm{dec}}, k^{\mathrm{dec}}, n^{\mathrm{dec}}\right)$. Clearly, the formulation does not depend on the parameters and holds always since the purpose of centralization is to maximize the entire SC profit but the decentralized model focuses on maximizing each SC participant, with no attention to the entire SC profit. 
Proof of Corollary 3.5. In the considered SC, under the centralized structure, each SC actor will not be allowed to maximize its profitability individually. While, the review period and safety factor in the decentralization $\left(T^{\text {dec }}\right.$ and $k^{\mathrm{dec}}$ ) are achieved in such a way to maximize the retailer's profit, considering $T^{\mathrm{dec}}$ and $k^{\mathrm{dec}}$ in comparison with $T^{\mathrm{cen}}$ and $k^{\mathrm{cen}}$, yields more benefit for the retailer. This result can be demonstrated as $\pi_{r}^{\text {dec }}\left(T^{\mathrm{dec}}, k^{\mathrm{dec}}\right) \geq$ $\pi_{r}^{\mathrm{cen}}\left(T^{\mathrm{cen}}, k^{\mathrm{cen}}\right)$. Achieving this formulation in our investigated SC is always true with no dependency on the parameters. The centralized setting overlooks each SC member's profitability. Based on Corollary 1, though the integrated setting yields more profit for the overall SC, it does not mean that all the SC participants will benefit from the centralization. In this study, the retailer will not accept the centralized model due to the losses incurred. Accordingly, to ensure the participation of all chain actors in the centralized setting, we need to propose an incentive scheme ensuring all SC members' profitability along with the entire SC.

Acknowledgements. The authors sincerely thank the editor, associate editor and reviewers for their kind and constructive comments and suggestions, which significantly improved this paper. This research did not receive any specific grant from funding agencies in the public, commercial, or not-for-profit sectors.

\section{REFERENCES}

[1] V. Albino, L. Fraccascia and I. Giannoccaro, Exploring the role of contracts to support the emergence of self-organized industrial symbiosis networks: an agent-based simulation study. J. Cleaner Prod. 112 (2016) 4353-4366.

[2] K. Annadurai and R. Uthayakumar, Reducing lost-sales rate in $(T, R, L)$ inventory model with controllable lead time. Appl. Math. Model. 34 (2010) 3465-3477.

[3] G.P. Cachon, Supply chain coordination with contracts. Handbooks Oper. Res. Manage. Sci. 11 (2003) $227-339$.

[4] G.P. Cachon and A.G. Kök, Competing manufacturers in a retail supply chain: On contractual form and coordination. Manage. Sci. 56 (2010) 571-589.

[5] L.E. Cárdenas-Barrón, The economic production quantity (EPQ) with shortage derived algebraically. Int. J. Prod. Econ. 70 (2001) 289-292.

[6] L.E. Cárdenas-Barrón and S.S. Sana, A production-inventory model for a two-echelon supply chain when demand is dependent on sales teamsx initiatives. Int. J. Prod. Econ. 155 (2014) 249-258.

[7] L.E. Cárdenas-Barrón, A.A. Shaikh, S. Tiwari and G. Treviño-Garza, An EOQ inventory model with nonlinear stock dependent holding cost, nonlinear stock dependent demand and trade credit. Comput. Ind. Eng. 139 (2018) 105557.

[8] S.K. Chaharsooghi and J. Heydari, Supply chain coordination for the joint determination of order quantity and reorder point using credit option. Eur. J. Oper. Res. 204 (2010) 86-95.

[9] T.M. Choi, Pricing and branding for remanufactured fashion products. J. Cleaner Prod. 165 (2017) 1385-1394.

[10] T.M. Choi, Y. Li and L. Xu, Channel leadership, performance and coordination in closed loop supply chains. Int. J. Prod. Econ. 146 (2013) 371-380.

[11] S. Chopra and P. Meindl, Supply chain management. Strategy, planning \& operation. In: Das Summa Summarum des Management. Gabler (2007) 265-275.

[12] A.P. de Barros, C.S. Ishikiriyama, R.C. Peres and C.F.S. Gomes, Processes and benefits of the application of information technology in supply chain management: an analysis of the literature. Proc. Comput. Sci. 55 (2015) 698-705.

[13] S. Ebrahimi, S.M. Hosseini-Motlagh and M. Nematollahi, Proposing a delay in payment contract for coordinating a two-echelon periodic review supply chain with stochastic promotional effort dependent demand. Int. J. Mach. Learning Cybern. 10 (2019) 1037-1050.

[14] P. Garcia-Herreros, A. Agarwal, J.M. Wassick and I.E. Grossmann, Optimizing inventory policies in process networks under uncertainty. Comput. Chem. Eng. 92 (2016) 256-272.

[15] I. Giannoccaro and P. Pontrandolfo, Negotiation of the revenue sharing contract: an agent-based systems approach. Int. J. Prod. Econ. 122 (2009) 558-566.

[16] B.C. Giri and B.R. Sarker, Coordinating a two-echelon supply chain under production disruption when retailers compete with price and service level. Oper. Res. 16 (2016) 71-88.

[17] K. Govindan and A. Malomfalean, A framework for evaluation of supply chain coordination by contracts under O2O environment. Int. J. Prod. Econ. 215 (2019) 11-23.

[18] K. Govindan, M.N. Popiuc and A. Diabat, Overview of coordination contracts within forward and reverse supply chains. J. Cleaner Prod. 47 (2013) 319-334.

[19] S. Guo, B. Shen, T.M. Choi and S. Jung, A review on supply chain contracts in reverse logistics: supply chain structures and channel leaderships. J. Cleaner Prod. 144 (2017) 387-402.

[20] J. Heydari, Supply chain coordination using time-based temporary price discounts. Comput. Ind. Eng. 75 (2014) 96-101.

[21] J. Heydari, M. Mahmoodi and A.A. Taleizadeh, Lead time aggregation: a three-echelon supply chain model. Transp. Res. Part E: Logistics Transp. Rev. 89 (2016) 215-233. 
[22] S.M. Hosseini-Motlagh, S. Ebrahimi and R. Zirakpourdehkordi, Coordination of dual-function acquisition price and corporate social responsibility in a sustainable closed-loop supply chain. J. Cleaner Prod. 251 (2020) 119629.

[23] S.M. Hosseini-Motlagh, M. Nouri, M. Johari and B.R. Sarker, Coordinating economic incentives, customer service and pricing decisions in a competitive closed-loop supply chain. J. Cleaner Prod. 255 (2020) 120241.

[24] M. Jazinaninejad, S.M. Seyedhosseini, S.M. Hosseini-Motlagh and M. Nematollahi, Coordinated decision-making on manufacturer's EPQ-based and buyer's period review inventory policies with stochastic price-sensitive demand: a credit option approach. RAIRO:OR 53 (2019) 1129-1154.

[25] M. Johari and S.M. Hosseini-Motlagh, Coordination contract for a competitive pharmaceutical supply chain considering corporate social responsibility and pricing decisions. RAIRO:OR 54 (2020) 1515-1535.

[26] M. Johari, S.M. Hosseini-Motlagh and M. Nematollahi, Simultaneous coordination of review period and order-up-to-level in a manufacturer-retailer chain. J. Ind. Syst. Eng. 10 (2017) 1-17.

[27] M. Johari, S.M. Hosseini-Motlagh, M. Nematollahi, M. Goh and J. Ignatius, Bi-level credit period coordination for periodic review inventory system with price-credit dependent demand under time value of money. Transp. Res. Part E: Logistics Trans. Rev. 114 (2018) 270-291.

[28] K. Kanchanasuntorn and A. Techanitisawad, An approximate periodic model for fixed-life perishable products in a two-echelon inventory-distribution system. Int. J. Prod. Econ. 100 (2006) 101-115.

[29] M. Karimi-Nasab and I. Konstantaras, An inventory control model with stochastic review interval and special sale offer. Eur. J. Oper. Res. 227 (2013) 81-87.

[30] C. Kouki and O. Jouini, On the effect of lifetime variability on the performance of inventory systems. Int. J. Prod. Econ. 167 (2015) 23-34

[31] J. Li and L. Liu, Supply chain coordination with quantity discount policy. Int. J. Prod. Econ. 101 (2006) 89-98.

[32] Q. Meng, Z. Li, H. Liu and J. Chen, Agent-based simulation of competitive performance for supply chains based on combined contracts. Int. J. Prod. Econ. 193 (2017) 663-676.

[33] N.M. Modak, S. Panda, S.S. Sana and M. Basu, Corporate social responsibility, coordination and profit distribution in a dual-channel supply chain. Pac. Sci. Rev. 16 (2014) 235-249.

[34] D.C. Montgomery, M.S. Bazaraa and A.K. Keswani, Inventory models with a mixture of backorders and lost sales. Nav. Res. Logistics Q. 20 (1973) 255-263.

[35] C.L. Munson and M.J. Rosenblatt, Theories and realities of quantity discounts: An exploratory study. Prod. Oper. Manage. 7 (1998) 352-369.

[36] M. Nematollahi, S.M. Hosseini-Motlagh and J. Heydari, Economic and social collaborative decision-making on visit interval and service level in a two-echelon pharmaceutical supply chain. J. Cleaner Prod. 142 (2017) 3956-3969.

[37] M. Nematollahi, S.M. Hosseini-Motlagh and J. Heydari, Coordination of social responsibility and order quantity in a twoechelon supply chain: a collaborative decision-making perspective. Int. J. Prod. Econ. 184 (2017) 107-121.

[38] M. Nematollahi, S.M. Hosseini-Motlagh, J. Ignatius, M. Goh and M.S. Nia, Coordinating a socially responsible pharmaceutical supply chain under periodic review replenishment policies. J. Cleaner Prod. 172 (2018) 2876-2891.

[39] J. Ni, J. Zhao and L.K. Chu, Supply contracting and process innovation in a dynamic supply chain with information asymmetry. Eur. J. Oper. Res. 288 (2021) 552-562.

[40] M. Nouri, S.M. Hosseini-Motlagh and M. Nematollahi, Proposing a discount policy for two-level supply chain coordination with periodic review replenishment and promotional efforts decisions. Oper. Res. (2018) 1-34.

[41] M. Nouri, S.M. Hosseini-Motlagh, M. Nematollahi and B.R. Sarker, Coordinating manufacturer's innovation and retailer's promotion and replenishment using a compensation-based wholesale price contract. Int. J. Prod. Econ. 198 (2018) 11-24.

[42] L.Y. Ouyang and B.R. Chuang, A periodic review inventory model involving variable lead time with a service level constraint. Int. J. Syst. Sci. 31 (2000) 1209-1215.

[43] L.Y. Ouyang and B.R. Chuang, A periodic review inventory-control system with variable lead time. Int. J. Inf. Manage. Sci. 12 (2001) 1-14.

[44] S. Panda, N.M. Modak and D. Pradhan, Corporate social responsibility, channel coordination and profit division in a twoechelon supply chain. Int. J. Manage. Sci. Eng. Manage. 11 (2016) 22-33.

[45] N. Privett and D. Gonsalvez, The top ten global health supply chain issues: perspectives from the field. Oper. Res. Health Care 3 (2014) 226-230.

[46] M.J. Rosenblatt and H.L. Lee, Improving profitability with quantity discounts under fixed demand. IIE Trans. 17 (1985) 388-395.

[47] B. Sarkar and A.S. Mahapatra, Periodic review fuzzy inventory model with variable lead time and fuzzy demand. Int. Trans. Oper. Res. 24 (2017) 1197-1227.

[48] E.A. Silver and D.P. Bischak, The exact fill rate in a periodic review base stock system under normally distributed demand. Omega 39 (2011) 346-349.

[49] D.P. Song, J.X. Dong and J. Xu, Integrated inventory management and supplier base reduction in a supply chain with multiple uncertainties. Eur. J. Oper. Res. 232 (2014) 522-536.

[50] H.N. Soni and M. Joshi, A periodic review inventory model with controllable lead time and backorder rate in fuzzy-stochastic environment. Fuzzy Inf. Eng. 7 (2015) 101-114.

[51] G. Tagaras and D. Vlachos, A periodic review inventory system with emergency replenishments. Manage. Sci. 47 (2001) $415-429$.

[52] Z.K. Weng, Channel coordination and quantity discounts. Manage. Sci. 41 (1995) 1509-1522. 
[53] R. Yang, W. Tang, R. Dai and J. Zhang, Contract design in reverse recycling supply chain with waste cooking oil under asymmetric cost information. J. Cleaner Prod. 201 (2018) 61-77.

[54] D.Q. Yao, X. Yue and J. Liu, Vertical cost information sharing in a supply chain with value-adding retailers. Omega 36 (2008) $838-851$.

[55] S. Yin, T. Nishi and I.E. Grossmann, Optimal quantity discount coordination for supply chain optimization with one manufacturer and multiple suppliers under demand uncertainty. Int. J. Adv. Manuf. Technol. 76 (2015) 1173-1184.

[56] Q. Zhang, J. Luo and Y. Duan, Buyer-vendor coordination for fixed lifetime product with quantity discount under finite production rate. Int. J. Syst. Sci. 47 (2016) 821-834.

[57] P. Zhang, Y. He and C.V. Shi, Transshipment and coordination in a two-echelon supply chain. RAIRO:OR 51 (2017) $729-747$. 